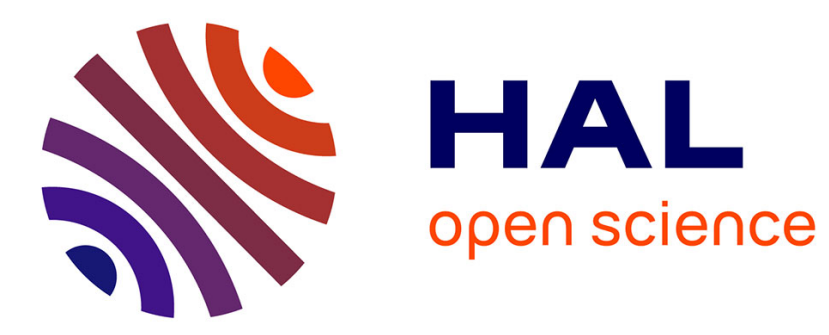

\title{
Impact of aligned carbon nanotubes array on the magnetostatic isolation of closely packed ferromagnetic nanoparticles
}

\author{
A.L. L Danilyuk, A.V. V Kukharev, Costel Sorin Cojocaru, F. Le Normand, \\ S.L. L Prischepa
}

\section{To cite this version:}

A.L. L Danilyuk, A.V. V Kukharev, Costel Sorin Cojocaru, F. Le Normand, S.L. L Prischepa. Impact of aligned carbon nanotubes array on the magnetostatic isolation of closely packed ferromagnetic nanoparticles. Carbon, 2018, 139, pp.1104-1116. 10.1016/j.carbon.2018.08.024 . hal-02325418

\section{HAL Id: hal-02325418 \\ https://hal.science/hal-02325418}

Submitted on 24 Oct 2019

HAL is a multi-disciplinary open access archive for the deposit and dissemination of scientific research documents, whether they are published or not. The documents may come from teaching and research institutions in France or abroad, or from public or private research centers.
L'archive ouverte pluridisciplinaire HAL, est destinée au dépôt et à la diffusion de documents scientifiques de niveau recherche, publiés ou non, émanant des établissements d'enseignement et de recherche français ou étrangers, des laboratoires publics ou privés. 


\title{
Impact of aligned carbon nanotubes array on the magnetostatic isolation of closely packed ferromagnetic nanoparticles
}

\author{
A.L. Danilyuk ${ }^{a}$, A.V. Kukharev ${ }^{a}$, C.S.Cojocaru ${ }^{\text {b, } 1}$, F. Le Normand ${ }^{\text {b, } 2}$, S.L.Prischepa ${ }^{\text {a, c, * }}$ \\ ${ }^{a}$ Belarusian State University of Informatics and Radioelectronics, P.Browka 6, Minsk, 220013, Belarus \\ ${ }^{\mathrm{b}}$ Institut de Physique et Chimie des Materiaux (IPCMS), CNRS-University of StrasbourgBP 43,67034, Strasbourg Cedex 2,France \\ ${ }^{\mathrm{c}}$ National Research Nuclear University (MEPhl),Kashirskoe Highway 31,Moscow, 115409,Russia
}

We investigate the influence of carbon nanotubes (CNT) aligned array on the magnetic properties of ensemble of densely packed Co nanoparticles (NPs) embedded inside CNT. Each CNT contains only one nanosized Co. Such a special structure was formed by catalyst chemical vapor deposition (CCVD) activated by current discharge plasma and hot filament. The Co NPs, previously deposited onto $\mathrm{SiO}{ }_{2} / \mathrm{Si}$ substrate, acted as a catalyst. By varying the parameters of the CCVD process, we were able to also sputter the substrate instead of CNT growth. Co NPs were used as a mask and the structure of Si-based nanocones with Co NPs on the top of each cone was formed. Exhaustive investigation of the structural, morphology and crystalline properties of Co nanoparticles were performed. The magnetic properties of two kinds of samples, Co on the Si-based nanocone and Co inside CNT, were differ drastically. In the former case, the magnetic anisotropy of thin-film-type has been observed with large magnetic domains. Whereas for the Co-CNT samples ferromagnetic NPs were magnetically isolated. It was established that the magnetic anisotropy of nanosized Co plays more dominant role than the dipole interaction between Co NPs. The role of the CNT container in this is discussed.

\section{Introduction}

At present, it has been unequivocally established that the properties of magnetic nanoparticles (NPs) differ significantly from bulk phase. For example, when the ratio of surface spins to the total number of NP's spins increases, surface effects dominate the overall NP properties [1]. Considering an ensemble of NPs it is worth underlying the interplay between interparticle direct or indirect exchange coupling and magnetic anisotropy, magnetic anisotropy and dipole-dipole interparticle interaction, intraparticle exchange coupling between surface and core. In other words, the interplay between collective effects and internal NP features determines the macroscopic properties of the whole system. In particular, in aggregated magnetic NPs, when they are in close contact, exchange

\footnotetext{
* Corresponding author. Belarusian State University of Informatics and Radioelectronics, P.Browka 6, Minsk, 220013 Belarus.

E-mail address: prischepa@bsuir.by (S.L.Prischepa).

1 Present address: Laboratoire de Physique des Interfaces et des Couches Minces, CNRS,Ecole Polytechnique, Universit e Paris Saclay,91128, Palaiseau Cedex,France.

2 Present address ICube/MaCEPV Laboratory, UMR 7357 CNRS-University of Strasbourg, BP20CR,67037 Strasbourg Cedex, France.
}

interactions could determine their characteristics. On the other hand, strong dipole-dipole interaction (DDI) between closely packed ferromagnetic NPs leads to collective effects [2e4]. However, for many applications, an ensemble of noninteracting magnetic NPs is required.

There are numerous approaches to create an ensemble of isolated (non-interacting) magnetic NPs. A variety of parameters which must be controlled, like NP parameters (chemical nature, distribution, mean size, concentration, interparticle spacing, shape, agglomeration), matrix material parameters (chemical nature, conductivity, permeability, method of NPs embedding into the matrix) create a huge space for experimental research in this interdisciplinary field [2,5e15]. The general tendency is that the increase of the interparticle spacing reduces the interparticle dipolar coupling and ultimately creates a system of non-interacting NPs $[16,17]$. A serious problem arising in the synthesis process however is agglomeration of nanoparticles. Various chemical methods of their fabrication, such as co-precipitation, thermal decomposition, hydrothermal, sol-gel and other approaches $[13,18]$ can only change the effectiveness of the agglomeration but cannot avoid it completely. Moreover, the agglomeration prevents the 
fabrication of an ensemble of closely packed magnetically isolated NPs, which is a great challenge for modern magnetoelectronics and spintronics.

Recently it has been demonstrated that carbon nanotubes (CNT) decorated by ferromagnetic nanoparticles can provide a very useful platform for studying various types of interparticle interaction, as well as impact of the CNT matrix on the nanoparticle's arrangement and interplay between individual and collective properties $[6,19]$. Indeed, when one produces oriented CNT arrays by chemical vapor deposition using $3 \mathrm{~d}$ metals ( $\mathrm{Fe}, \mathrm{Ni}, \mathrm{Co}$ ) as a catalyst (CCVD), such samples can be considered as a magnetic nanocomposite with ferromagnetic NPs embedded inside CNT matrix. By floating CCVD, depending on the concentration of the catalyst, one can get ferromagnetic NPs located only inside the inner CNT channels for low concentration, or both distributed inside and outside the CNT walls for high catalyst concentration $[19,20]$. In the latter case NPs are strongly coupled via direct and indirect exchange interaction [21e23], whereas in the former case there are indications of magnetically isolated NPs [24]. This method creates nanotubes with many ferromagnetic NPs inside the inner channel of the same nanotube and the mean distance between adjacent nanoparticles is order of hundreds of nanometers [23]. Nevertheless, the characteristic feature of the exchange coupling between these NPs was observed $[21 \mathrm{e} 23,25]$. It was suggested that due to high spin orbit interaction in defective CNTs the indirect exchange coupling via CNT inner shell is the reason for this $[23,26]$.

It is natural to expect that if each CNT contains only one ferromagnetic nanoparticle inside an inner channel and all CNTs are perfectly aligned, but are not in contact with each other, then one can get an ensemble of densely packed ferromagnetic NPs without the possibility of any exchange coupling between them. Such kind of samples can be synthesized by the plasma-enhanced CCVD (PECCVD) process on flat substrate onto which NPs of a $3 d$ metal were preliminary deposited. The PE-CCVD growth is activated by a direct current discharge plasma (PE CCVD) or by heating by hot filament (HF CCVD), or by both processes (PE HF CCVD). As it was demonstrated in Refs. [27,28], application of both modes of gas activation is the best adapted to grow vertically aligned carbon nanotubes using Co NPs as a catalyst. However, with a high carbon concentration in the PE HF CCVD mode, competition between carbon deposition and substrate sputtering may occur [27]. Under appropriate conditions, it is then possible to grow new highly oriented materials with a single individual ferromagnetic Co NP on top.

In this paper, we consider nanomaterials synthesized by PE HF CCVD on SiQ/Si substrates covered with Co NPs at different carbon concentration during the growth process. We get quite different configurations of Co NPs and we investigate their magnetic properties. After this Introduction, the paper is organized in 3 sections: the experimental preparation procedure and sample characterization are described in Section 2. We underline especially that, depending on the parameters of the PE HF CCVD, it is possible to obtain a single Co nanoparticle embedded on top of a silicon oxynitride $\left(\mathrm{SiN}{ }_{x} \mathrm{O}_{y}\right.$ ) nanocone and a single Co quasi-nanocylinder inserted inside CNTs. By means of electron diffraction we prove the existence of both fcc and hcp Co. The competition between intrinsic and long-range DDI is experimentally investigated in Section 3. For that we start from magnetic force microscopy (MFM) measurements, afterwards we analyze field cooled and zero field cooled (FC-ZFC) temperature dependence of the magnetization. From both results we unambiguously obtain that for Co-CNT samples inclusions are non-interacting while in the Co-SiN $x \mathrm{O}_{y}$ case the DDI between Co nanoparticles dominates. Then the magnetization $M(H)$ loops are investigated. To minimize the effect of temperature, data were acquired at $\mathrm{T} 1 / 42 \mathrm{~K}$. We show that for $\mathrm{Co}-\mathrm{SiO}_{\mathrm{x}} \mathrm{N}_{\mathrm{y}}$ samples the in-plane magnetic anisotropy dominates, which confirms strong DDI within the plane. Reversely for Co-CNT samples, it is not possible to distinguish the easy axis of magnetization. In Section 4 both magnetic systems are studied and the micromagnetic parameters including the effective magnetic anisotropy constant of Co NPs are estimated within the random anisotropy model (RAM). We show that the field of the random anisotropy exceeds the exchange field in both cases. But the nature of the magnetic anisotropy contribution is still unclear. To solve this problem, we consider the most significant contributions to the obtained effective magnetic anisotropy constant of Co NPs. For that we perform a comparative analysis of the DDI and magnetic anisotropy and we deduce the contribution of the DDI, magnetocrystalline, magnetoelastic and shape anisotropies (MCA, MEA and SA, respectively). We underline that, the contribution of the MEA depends essentially on the type of the crystalline lattice. We separately estimate the contribution of fcc and hcp Co to the elastic stresses. In the latter case different orientations of the hexagonal axis with respect to the CNT alignment are considered. It is obtained that maximum elastic stresses occur when the hexagonal axis is oriented perpendicular to the CNT axis. Even within these conditions Co remains elastic and the corresponding deformations of the CNT lattice do not exceed $2 \%$. At the end of this section, we show the results of the micromagnetic simulations which support the idea about the perpendicular orientation of the hexagonal axis of hcp Co. The article ends with some concluding remarks and outlook.

\section{Experimental and Co NP's characterizations}

$\mathrm{A} \mathrm{SiO}_{2}$ layer of $5 \mathrm{~nm}$ thickness was first deposited onto $\mathrm{Si}(100)$ substrate by an electron cyclotron resonance plasma process. The two samples preparation are then processed differently.

\subsection{Nanoparticles on top of nanocones: synthesis and characterization}

An ultrathin Co film $(2.5 \mathrm{~nm})$ was sputtered by DC magnetron. The buffer $\mathrm{SiO}_{2}$ layer prevents the formation of Co silicide. In addition, there is large difference in surface energies of $\mathrm{SiO}_{2}$ and $\mathrm{Co}$ which facilitates the coalescence of former Co atoms with the formation of small nanoparticles. Then the substrate was transferred into an UHV CVD reactor and softly elaborated by slow thermal reaction up to $973 \mathrm{~K}$ with a heat rate of $10 \mathrm{~K} / \mathrm{min}$ under UHV and above $673 \mathrm{~K}$ under a hydrogen/ammonia (80:20) gas flow at 15 mbar. Finally, the metal reduction and the formation of Co NPs were performed in a thermally and plasma-activated mixture of hydrogen and ammonia at $973 \mathrm{~K}$ during $15 \mathrm{~min}$. As a result, an ensemble of Co NPs was synthesized on the surface of $\mathrm{SiO}_{2} / \mathrm{Si}$ substrates. Then, the process of PE HF CCVD was performed in the mixture of $\mathrm{C}_{2} \mathrm{H}_{2}: \mathrm{NH}_{3}: \mathrm{H}_{2} \quad 1 / 4$ 20:16:64 with a flow rate $D$ flow $1 / 4100$ sccm at 15 mbar. The conditions are summarized in Table 1 for Co catalyst preparation and in Table 2 for nanostructures preparation. As a result, in the presence of heavy nitrogen-based ion, sputtering of the $\mathrm{SiO}_{2} / \mathrm{Si}$ substrates readily occurs. Therefore, the resulting sample consists of silicon oxynitride nanocones with the Co NPs merging on the top of each nanocone. However, the Co NPs behaved like a mask during the sputtering process, protecting the area below from sputtering. Also, the sputtering by directional ions allowed the definition of geometrical nanocones centered around the Co NP mask. A SEM image (XL30S-FEG PHILIPPS working at $3 \mathrm{KV}$ ) of a typical sample is shown in Fig. 1a and a TEM image (TOPCON 002B working at $200 \mathrm{kV}$ with dry surface scratching) is displayed in Fig. $1 \mathrm{~b}$.

Other synthesis conditions (experimental set-up, heating and cooling steps, plasma activation parameters) and Co NPs properties (size histogram and SEM image in top view) are displayed in the 
Table 1

Elaboration conditions of the Co catalysts. $T_{f}$ is the temperature of filaments.

\begin{tabular}{|c|c|c|c|c|c|c|c|c|}
\hline \multirow[t]{2}{*}{ Deposit } & \multirow[t]{2}{*}{ Nature } & \multirow[t]{2}{*}{$\begin{array}{l}\text { Catalyst deposition } \\
\text { mode }\end{array}$} & \multirow[t]{2}{*}{ Pressure (mbar) } & \multirow[t]{2}{*}{$\begin{array}{l}\text { Temperature } \\
(\mathrm{K})\end{array}$} & $\begin{array}{l}\text { Catalyst } \\
\text { reduction }\end{array}$ & \multirow[t]{2}{*}{$\begin{array}{l}\text { Temperature rate }(\mathrm{K} / \\
\min )\end{array}$} & \multirow[t]{2}{*}{$\begin{array}{l}\text { Power filaments }(\mathrm{W})\left[\mathrm{T}_{\mathrm{f}}\right] \text { (above } \\
573 \mathrm{~K})\end{array}$} & \multirow[t]{2}{*}{ Plasma } \\
\hline & & & & & $\begin{array}{l}\mathrm{C}_{2} \mathrm{H}_{2}: \mathrm{NH}_{3}: \\
(\%)\end{array}$ & & & \\
\hline \multicolumn{2}{|c|}{$\begin{array}{l}\text { Nanocones } \\
\text { Silicon } \\
\text { oxynitride }\end{array}$} & Sputtering & \multicolumn{2}{|c|}{ UHV(573K) p 15973} & $0: 20: 80$ & 10 & $150[2100 \mathrm{~K}]$ & Yes \\
\hline \multicolumn{2}{|c|}{ Nanotubes Carbon } & Evaporation & UHV(973K) p 15 & 973 & $0: 0: 100$ & 10 & $150[2100 \mathrm{~K}]$ & No \\
\hline
\end{tabular}

Table 2

Elaboration conditions of the nanostructures by PE HF CCVD. For plasma conditions see SI-1.

\begin{tabular}{|c|c|c|c|c|c|c|c|}
\hline Deposit & Nature & Pressure (mbar) & Temperature (K) & $\mathrm{C}_{2} \mathrm{H}_{2}: \mathrm{NH}_{3}: \mathrm{H}_{2}(\%)$ & $\mathrm{D}_{\text {flow }}(\mathrm{sccm})$ & $\mathrm{t}(\min )$ & Power filaments $(\mathrm{W})\left[\begin{array}{ll}T_{f}\end{array}\right]$ \\
\hline Nanocones & Silicon oxynitride & 15 & 973 & $20: 16: 64$ & 100 & 15 & $150[2100 \mathrm{~K}]$ \\
\hline Nanotubes & Carbon & 15 & 973 & $20: 0: 80$ & 100 & 6 & $150[2100 \mathrm{~K}]$ \\
\hline
\end{tabular}

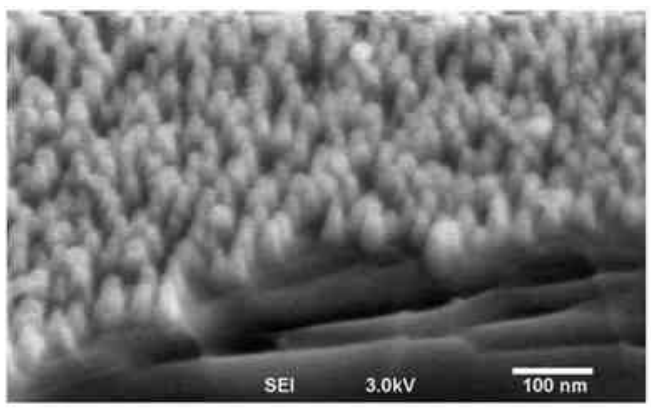

(a)

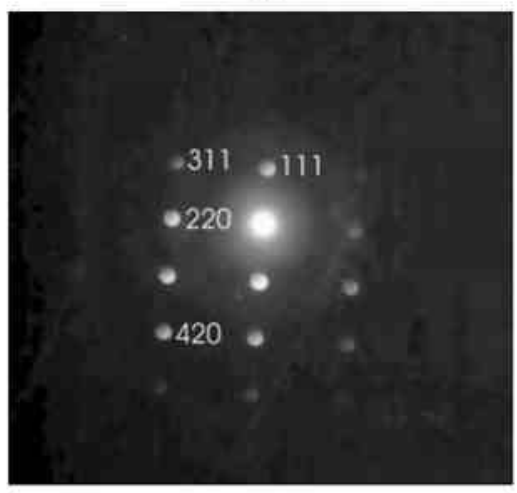

(c)

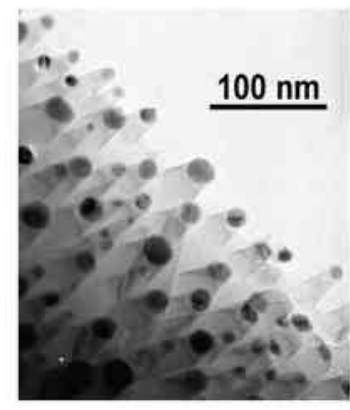

(b)

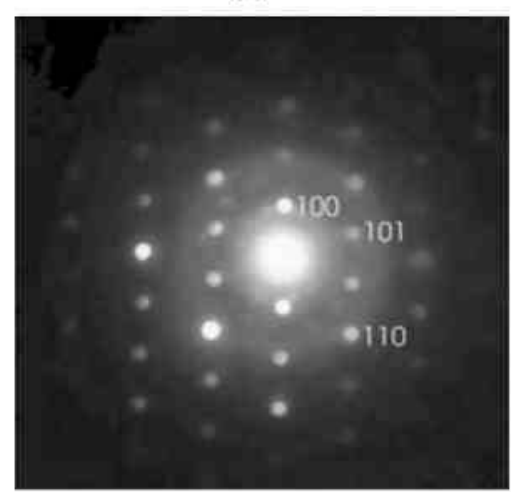

(d)

Fig. 1. SEM (a) and TEM (b) images of sample consisting of Co NPs on the top of Si oxynitride nanocones. Both images suggest aligned nanocones with a dark NP on top. Electron diffraction showing (c) cubic (fcc) and (d) hexagonal (hcp) lattices of Co NPs.

Supplementary Information 1 (SI 1) and 2 (SI 2), respectively. The size distribution is not completely monomodal with a main maximum at around $25 \mathrm{~nm}$ with secondary maxima around $40 \mathrm{~nm}$ and $13 \mathrm{~nm}$, respectively. The density is around $410{ }^{10} \mathrm{~cm}^{2}$. The nanoparticles are isotropic. Morphology and geometrical parameters of Co NPs are summarized in Table 3.
The crystalline structure of the NPs has been studied by TEM electron diffraction. The Co was observed with both cubic fcc (Fig. 1c) and hexagonal hcp (Fig. 1d) lattice. The fcc and hcp patterns assignation are reported in Supplementary Information 3 (SI 3 ) together with the corresponding ASTM files. NPs were mainly fcc structure however.

Table 3

Morphology and geometry of the nanosized Co.

\begin{tabular}{|c|c|c|c|c|}
\hline Deposit & Deposition process & Mean size $(\mathrm{nm})$ & Density $\left(10^{10} / \mathrm{cm}^{2}\right)$ & NP's shape \\
\hline Nanocones & $\mathrm{DC}$ magnetron $(2.5 \mathrm{~nm})$ & $25(40,13)$ & 4 & Isotropic \\
\hline Nanotubes & Evaporation (4.5 nm) & 13 & 1.3 & Anisotropic \\
\hline
\end{tabular}


The chemical nature of the nanocones was generally investigated by in situ X-Ray photoelectron spectroscopy (XPS) (nonmonochromatized $\mathrm{Mg} \mathrm{K}$ a source with VSW100 detector working at $0.65 \mathrm{eV}$ resolution) or ex situ analyzed (monochromatic Al Ka source with a VSW150 detector working at $\quad 0.55 \mathrm{eV}$ resolution). Without exposure to air, the sample is transferred in a surface analysis chamber directly connected to the deposition/treatment chamber. In Fig. 2 we show the survey XPS spectra of a sample cleaned by the $\mathrm{H}_{2}: \mathrm{NH}_{3}$ plasma before (bottom spectrum, red line) and after the $\mathrm{C}_{2} \mathrm{H}_{2}: \mathrm{NH}_{3}: \mathrm{H}_{2}$ exposition (upper curve, blue line) (i.e. before and after synthesis of nanocones with Co NPs on top). Both spectra were recorded with an unmonochromatized MgK a source. The presence of silicon ( $\mathrm{Si} 2 \mathrm{p}$ and $\mathrm{Si} 2 \mathrm{~s})$, oxygen (O1s and O2s), nitrogen (N1s) and cobalt (Co2p and Co3p) on the surface is clearly evidenced. The most important effect is the presence of nitrogen, which indicates, together with the presence of oxygen, a pronounced plasma-enhanced CVD formation of silicon oxynitride ( $\left.\mathrm{SiN}_{\mathrm{y}} \mathrm{Ox}\right)$. Their intensities do not much change with the two successive treatments. Importantly, only a negligible contribution of carbon is present (around $5 \%$ with graphitic nature at $284.6 \mathrm{eV}$ ), despite the acetylene injection into the CVD reactor. Also, the nitrogen concentration becomes even stronger after the $\mathrm{C}_{2} \mathrm{H}_{2}: \mathrm{NH}_{3}: \mathrm{H}_{2}$ exposition. The individual spectra of the Co2p, Si2p, N1s and O1s core levels are reported in Supplementary Information 4 (SI 4) in two situations: either after reduction in UHV for Co2p line and after synthesis of nanocones for all of them. They are completed with Co2p core level spectrum after air removal followed with a UHV thermal annealing at $873 \mathrm{~K}$. All these spectra show the presence of a weak $\mathrm{CoO}$ contribution at $782.3 \mathrm{eV}$ even after synthesis, besides the main $\mathrm{Co}^{0}$ contribution at $777.8 \mathrm{eV}$.

The surface concentration extracted from the XPS Si2, C1s, N1s, 01s, Co2p3/2 corelines are summarized in Table 4 . It is seen that the main Si2p peak at $101.65 \mathrm{eV}$, the main N1s peak at $397.3 \mathrm{eV}$ and the main $\mathrm{O} 1 \mathrm{~s}$ peak at around $532 \mathrm{eV}$ can be associated with the silicon oxynitride ( $\mathrm{SiNO}$ 0.3) in good agreement with the XPS assignation reported on the surface oxidation of silicon nitride in the literature, with $\mathrm{Si} 2 \mathrm{p}, \mathrm{N} 1 \mathrm{~s}$ and O1s main peaks at 101.6, 397.6 and $532.7 \mathrm{eV}$, respectively [29]. The cobalt is mainly in a metallic form with a characteristic $\mathrm{Co}^{0} 2 \mathrm{p} 3 / 2$ contribution at $777.8 \mathrm{eV}$. The presence of some Co oxide (CoO) after the nanocones formation at $782.3 \mathrm{eV}$ would mean that metallic Co on top of $\mathrm{SiO}{ }_{x} \mathrm{~N}_{y}$ nanocone is covered

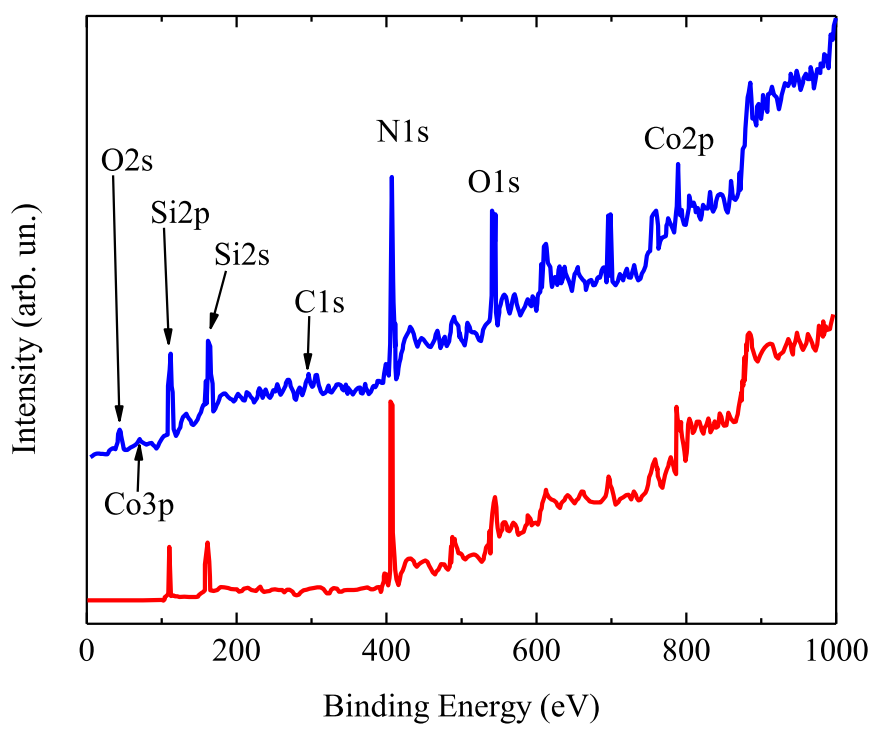

Fig. 2. XPS survey spectra before (bottom, red) and after (up, blue) synthesis of nanocones. In situ XPS analysis. (A colour version of this figure can be viewed online.) with some oxide and thus represents some core/shell structure. Finally, the absence of carbon at the surface is confirmed by the very weak Raman signal in the carbon range $\left(1100-1650 \mathrm{~cm}^{1}\right.$ ) (not shown here).

\subsection{Nanoparticles inside the CNT: synthesis and characterization}

In the second approach, the catalyst was deposited with an evaporation cell in an UHV chamber directly connected to the synthesis chamber ( $4.5 \mathrm{~nm}$ of equivalent Co layer). The use of UHV conditions (base pressure $10^{9}$ mbar) during the evaporation allows to finely control the metal dispersion as well as the metallic nature of the deposit. This is supported by the XPS spectra of wide scans before and after Co evaporation as well as by the Co2p core level reported in Fig. 3 . The Co2p3/2 line exhibits a single line with a binding energy $(777.8 \mathrm{eV})$ and an asymmetry of the line (with, according to a Doniach-Sunjic fitting analysis [30], a full width at halfmaximum $\mathrm{G} 1 / 40.6 \mathrm{eV}$ and an asymmetry factor $\mathrm{a} 1 / 40.37$ instead of $\mathrm{G}^{1} 1 / 40.61$ and $\mathrm{a} 1 / 40.375$ [31]) quite coherent with metallic cobalt exclusively (Fig. 3d). The absence of impurity as well as the full metallic nature of evaporated Co render easier the reduction step with a single temperature rise at $973 \mathrm{~K}$ at the rate of $10 \mathrm{~K} / \mathrm{min}$.

The PE HF CCVD carbon nanotube growth was carried out with a mixture of $\mathrm{C}_{2} \mathrm{H}_{2}$ and $\mathrm{H}_{2}$ (without ammonia) at $\mathrm{p} 1 / 415 \mathrm{mbar}$ for $6 \mathrm{~min}$. The conditions are summarized in Tables 1 and 2 . These conditions (temperature, heating up and down, etc.) were cjosen to optimize the formation of homogeneous distribution of NPs. In this case the substrate was not etched but a vertically aligned array of CNTs is formed $[27,28]$. Small time of synthesis was chosen to minimize the formation of defects in carbon nanotubes. The quality of the grown nanotubes has been checked by Raman spectroscopy (Renishaw apparatus with He-Ne laser source) (Fig. 4). The spectrum in the carbon domain exhibits weak $D$ lines split at $1306 \mathrm{~cm} 1$ and at $1334 \mathrm{~cm}^{1}$, respectively. By contrast the intense $\mathrm{G}$ line exhibits a Breit-Wigner-Fano (BWF) shape with the main contribution at $1591 \mathrm{~cm}^{1}$ with a small full width at half maximum (FWHM) of $13 \mathrm{~cm}^{1}$ and weaker downward vibration modes at $1555 \mathrm{~cm}^{1}$, together with the $D{ }^{0}$ mode at $1606 \mathrm{~cm}^{1}$. This spectrum strongly suggests that the carbon nanotubes are mainly conductive with a high quality. A very small contribution due to amorphous carbon at $1525 \mathrm{~cm}^{1}$ can be observed.

Further characterization of the samples has been done by atomic force microscopy (AFM: NANOSCAN Dimension 3100 used in the tapping mode with Si cantilever and lateral resolution $5 \mathrm{e} 10 \mathrm{~nm}$ ), SEM and TEM. These investigations strongly suggest the massive growth of vertically oriented nanotube (Fig. 5a). This is expected when using a plasma activated process [27]. Each CNT contains only one Co nanoparticle inserted and located at the top of the nanotube. The AFM image of the Co surface before CNT growth is presented in Fig. $6 a$ and its $3 \mathrm{D}$ reconstruction is depicted in Fig. 6b, while the size distribution of the Co NPs is shown in Fig. 6c. The diameter distribution is well described by the log-normal distribution function as shown in Fig. $6 \mathrm{c}$, with the average diameter around $13 \mathrm{~nm}$. The density of NPs was evaluated to be $1.310{ }^{10} \mathrm{~cm}^{2}$ (Table 3). The TEM result confirms the presence of Co inclusions on the top of each CNT (Fig. 5b). The average length of CNT array is around $1 \mathrm{~mm}$. It should be emphasized that the major part of these Co nanoparticles is anisotropic, as can be seen in Fig. $5 c$ and d, with the long axis always oriented parallel to the nanotube axis. Thus, in Fig. $5 d$ the length $L$ is around $70 \mathrm{~nm}$ and the revolution width $W$ is $20 \mathrm{e} 25 \mathrm{~nm}$, which yields an aspect ratio L/W of around 3 . Fig. 5c shows Co nanoparticles with an anisotropic nailhead form. This is the most characteristic feature of these samples as reported [32]. The diameter of these inclusions is restricted by the inner channel of carbon nanotube and their length can reach $250 \mathrm{e} 300 \mathrm{~nm}$, i.e. an 
Table 4

Concentration analysis from the XPS data before and after the nanocones (nanotubes) formation.

\begin{tabular}{|c|c|c|c|c|c|c|c|c|c|}
\hline \multirow[t]{2}{*}{ Sample } & \multirow[t]{2}{*}{ Assignment } & \multicolumn{2}{|l|}{$\mathrm{Si} 2 \mathrm{p}$} & \multirow{2}{*}{$\frac{\mathrm{N} 1 \mathrm{~s}}{\mathrm{SiO}_{x} \mathrm{~N}_{\mathrm{y}}}$} & \multicolumn{2}{|l|}{ O1s } & \multicolumn{2}{|l|}{$\mathrm{Co} 2 \mathrm{p}_{3 / 2}$} & \multirow[t]{2}{*}{$\mathrm{C} 1 \mathrm{~s}$} \\
\hline & & $\mathrm{Si}$ & $\mathrm{SiO}_{x} \mathrm{~N}_{\mathrm{y}}$ & & $\mathrm{O}_{\text {surface }}$ & $\mathrm{SiO}_{x} \mathrm{~N}_{\mathrm{y}}(\mathrm{pCoO})$ & Co & $\mathrm{CoO}$ & \\
\hline Before nanocone formation & Energy $(\mathrm{eV})$ [Concentration, \%] & $100.8[3]$ & $101.6[31]$ & $397.4[38]$ & $535.0[3]$ & $533.1[6]$ & $778.1[19]$ & e & e \\
\hline After nanocone formation & Energy $(\mathrm{eV})$ [Concentration, \%] & $\mathrm{e}$ & $101.65[35]$ & $397.3[35]$ & $533.6[2]$ & $531.65[10]$ & $778.1[12]$ & $782.6[1]$ & $284.6[5$ \\
\hline After nanotube formation & Energy $(\mathrm{eV})$ [Concentration, \%] & $\mathrm{e}$ & $102.3[9]$ & e & e & $533.6[16]$ & $777.9[3]$ & $\mathrm{e}$ & $284.6[72]$ \\
\hline
\end{tabular}
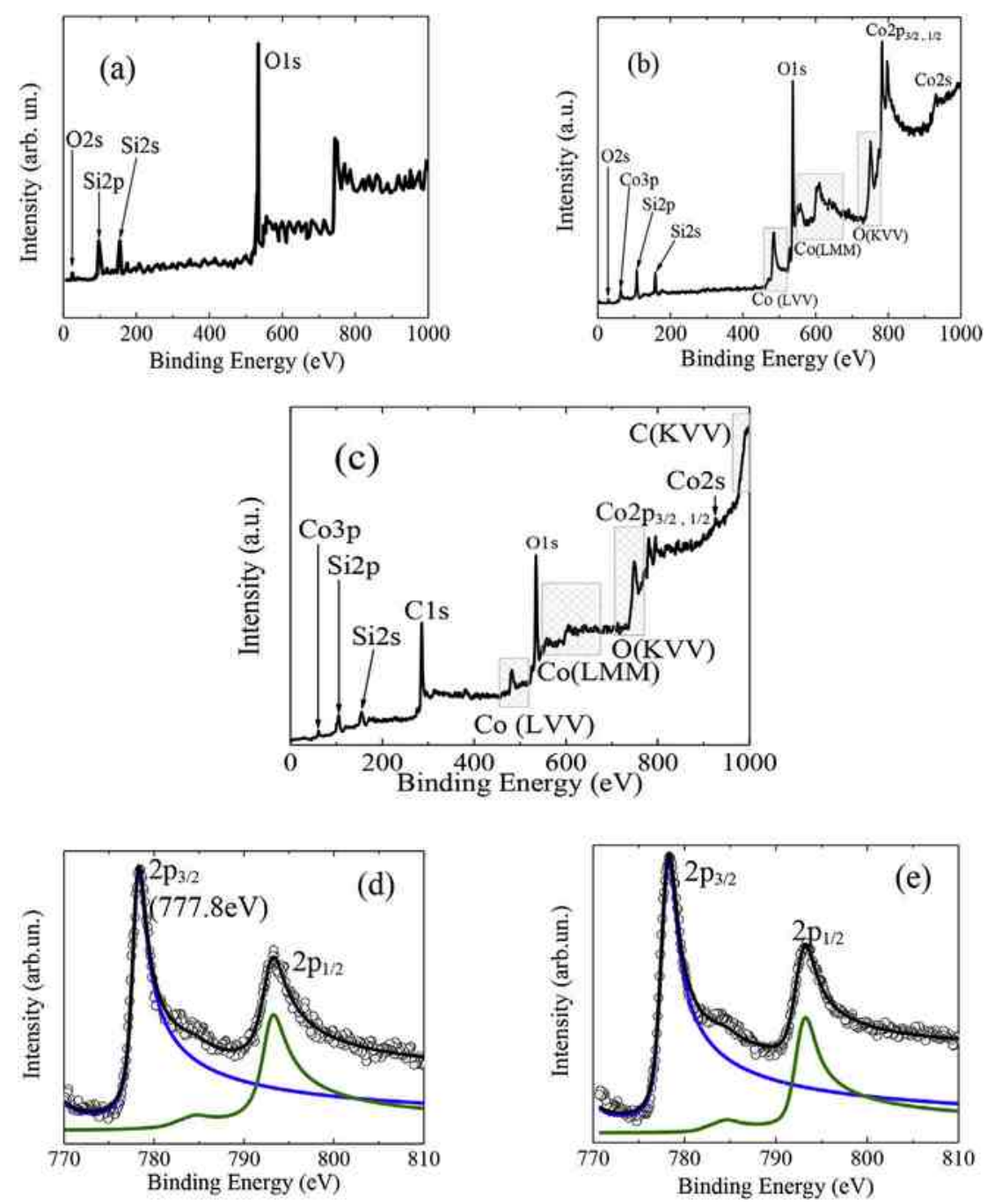

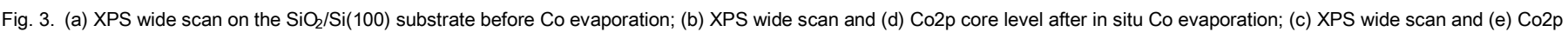

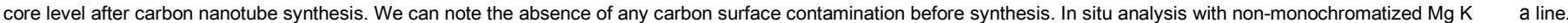
The Co $2 p 3 / 2$ and Co $2 p^{1 / 2}$ lines are fitted with two Doniach-Sunjic lines [30]. (A colour version of this figure can be viewed online.)

aspect ratio up to 20 . Such drastic change of a morphology of catalytic Co NPs during the CCVD of carbon nanotubes confirms the complicated nature of the growth process. The most likely process occurs through a top growth mode described elsewhere [32]. We can exclude the possible melting of Co due to relatively low temperature of the CCVD (973K). However, the nailhead shape of the Co nanoparticle often observed suggests that some diffusion occurs inferring nanoparticle shape transformation. This can be explained by large incorporation of carbon atoms driving the formation into some viscous state at a temperature not far from the eutectic temperature of the cobalt nanoparticle, an intermediate state between the solid or liquid state. Anyway, such resulting Co morphology embedded on top of CNT underlines the importance of diffusion processes in/on the NP bulk/surface during the CNT growth. TEM analysis reveal the regular structure of CNTs and their low defectiveness in agreement with the Raman spectroscopy. The nanotubes are multiwall but with a clear and regular hollow inside. More details about the processes of PE HF CCVD for vertically aligned CNT growth can be found elsewhere $[27,28,32,33]$.

\section{Magnetic properties}

\subsection{Magnetic force microscopy and FC-ZFC measurements}

In Fig. 7 we show the results of the AFM and magnetic force microscopy (MFM) recorded on NANOSCAN Dimension 3100 of 


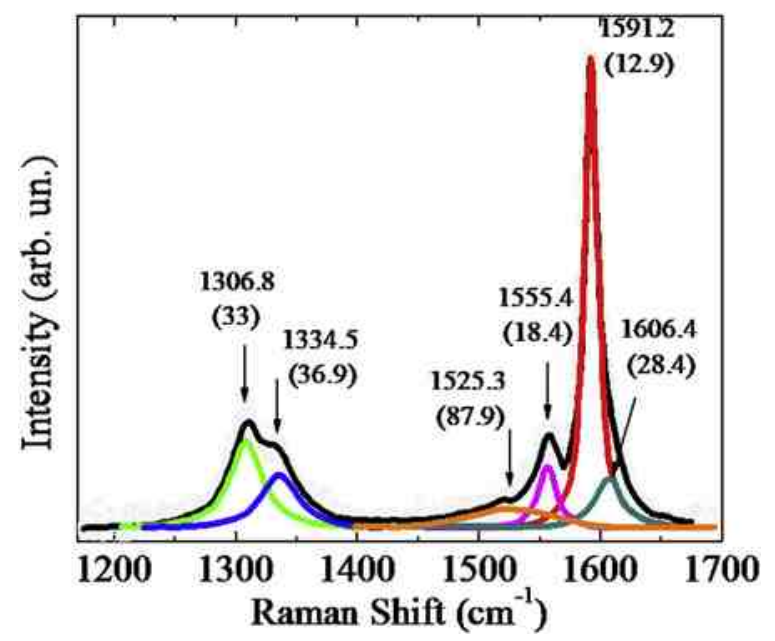

Fig. 4. Raman spectrum of arrays of vertically aligned CNT acquired with the laser wavelength of $632.8 \mathrm{~nm}$. Numbers indicate the Raman shift value for a peculiar mode. The numbers in brackets indicate the full width at half maximum in the same mode. Points are for the experimental data. Colored lines correspond to the different vibration modes specified in the text. The black line is the envelope obtained from all these considered modes. (A colour version of this figure can be viewed online.)

arrays of Co NPs at the top of silicon oxynitride nanocones. Sample was previously without magnetic history and both AFM and MFM images were acquired on the same part of the sample. To separate the short-range Van der Waals forces from the long-range magnetic forces, the same area has been scanned twice at distance $z$ (AFM image) and zpd, successively. The distance between the sample and cantilever was taken as $d 1 / 4500 \mathrm{~nm}$. Then the contribution of the magnetic force is determined from the phase variation which is proportional to the second derivative of the magnetic field. Despite the very rough character of the surface it is possible to detect by AFM the nanocones, with a size lower than $100 \mathrm{~nm}$. Moreover, by MFM it is possible to detect magnetic domains on the surface of the sample with average sizes around $500 \mathrm{~nm}$ encompassing a set of

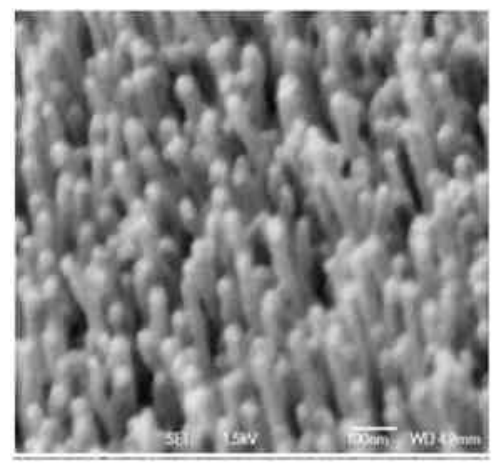

(a)

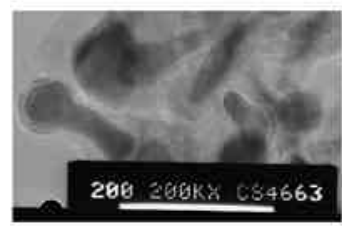

(c) many nanocones, which confirms the strong DDI between Co NPs. Other images are given in SI-5. It should be emphasized that these images were obtained on the sample without magnetic history, but we failed to record contrasted MFM images on the sample with a remanent magnetism after exposition to a magnetic field parallel or normal to the surface. This is likely due, as will be seen further, to

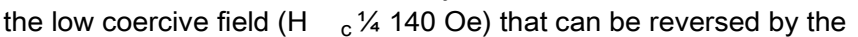
magnetic field of the cantilever in the former case, and to the very

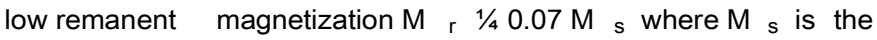
magnetization at saturation in the latter case.

In Fig. 8a we show the FC-ZFC magnetization curves for the same sample measured using a MPMS SQUID-VSM magnetometer (Quantum Design) combining the high speed of a VSM (vibrating sample magnetometer) with the sensitivity of the SQUID (Superconducting Quantum Interference Detector) magnetometer (MPMS). The magnetic field $\mathrm{H} 1 / 425$ Oe was oriented parallel to the surface substrate. The characteristic feature of the FC-ZFC curve is that up till $390 \mathrm{~K}$ the system never reaches the equilibrium state at which the field cooled magnetization, M FC becomes equal to zerofield cooled magnetization, MZFG Blocking temperature $T_{B}$ for the investigated Co NPs is close to $400 \mathrm{~K}$. It means that below this value nanoparticles are ferromagnetic with well-defined coercivity (not shown here). Importantly, the $\mathrm{M} \mathrm{Fd}(\mathrm{T})$ curve is almost temperatureindependent for $\mathrm{T}<400 \mathrm{~K}$. This is again an indication of strong DDI between magnetic NPs $[37,38]$. Thus, two independent measurement techniques confirm the presence of a strong DDI between the nanoparticles localized at the top of SiO ${ }_{x} \mathrm{~N}_{y}$ nanocones.

Now we move to the AFM-MFM and FC-ZFC magnetization results for the Co NPs localized inside CNT. In Fig. 9 AFM-MFM images are displayed for this sample. It is clearly seen that NPs are magnetically decoupled. It follows from the presented result that there is a unique correlation between the geometrical sizes of particles and the size of magnetic domains, - each particle corresponds to a single domain.

In Fig. $8 \mathrm{~b}$ we present the $\mathrm{M}_{\mathrm{FC}}$ and $\mathrm{M}_{\mathrm{ZFC}}$ plots versus temperature measured at $\mathrm{H}^{1} 1 / 425$ Oe for arrays of Co NPs embedded inside CNTs. Magnetic field was applied parallel to the substrate surface. The blocking temperature is around $400 \mathrm{~K}$, like for Co NPs on silicon

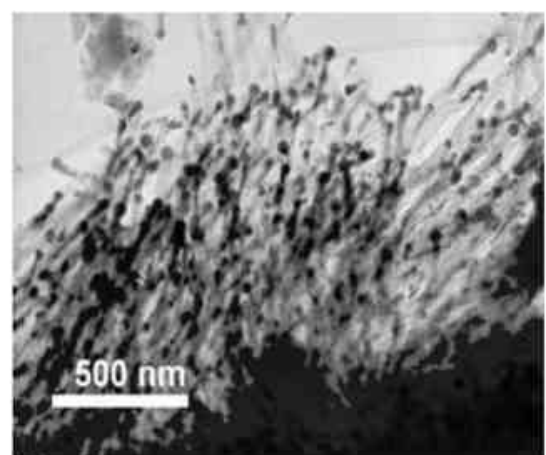

(b)

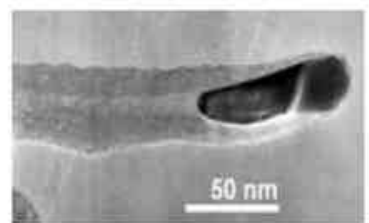

(d)

Fig. 5. (a) SEM and (b) TEM images of CNT grown on $\mathrm{SiO}_{2} / \mathrm{Si}$ substrate with Co NPs; (c) and (d) display the structural shape of the Co nanoparticles. 


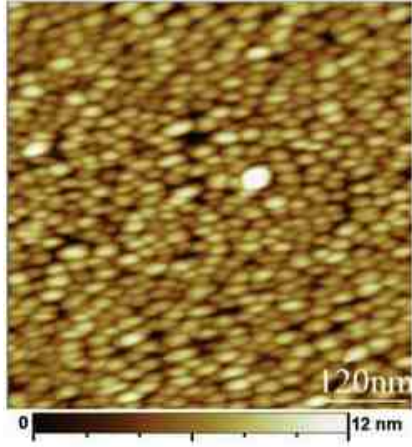

(a)

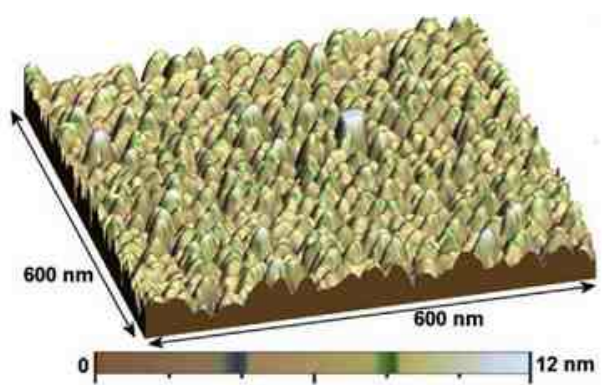

(b)

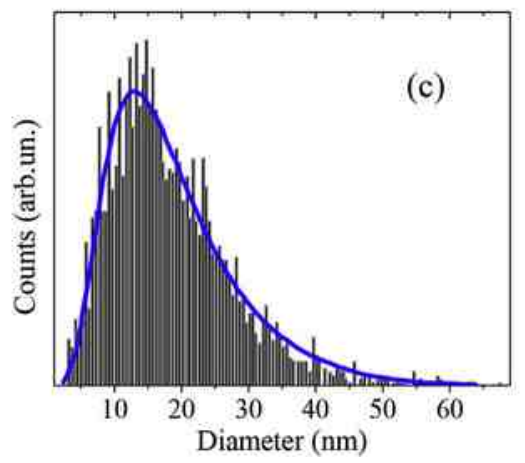

Fig. 6. (a) AFM image of Co NPs on $\mathrm{SiO}_{2} / \mathrm{Si}$ substrate; (b) Three-dimensional reconstruction of the same surface; (c) Distribution of the Co NP's size histogram (5000 nanoparticles) fitted with a log-normal law (blue curve) peaking at around $13 \mathrm{~nm}$.

(A colour version of this figure can be viewed online.)

oxynitride nanocones. Unlike the previous sample however, the $\mathrm{M}_{\mathrm{Fd}} \mathrm{T}$ ) behavior is quite different. Now it almost linearly increases when temperature goes to zero. This is characteristic feature of magnetically decoupled NPs $[4,11,34,35]$.

In conclusion, from the data presented in this section, it follows that the competition between the DDI and intrinsic properties of ensemble of Co NPs of approximately of the same diameter and the same density distribution varies drastically according to the preparation process. When NPs are localized on the top of silicon oxynitride nanocones, the DDI dominates. When NPs are embedded inside CNTs, the intrinsic interaction becomes dominant. This is a major result of this comparative work. In the following, we will try to elucidate why the DDI between Co inclusions becomes less dominant and explore the role of CNT in this.

\subsection{Analysis of the $M(H)$ loops}

Important information can be obtained from the analysis of the hysteresis loops $M(H)$. The measured $M(H)$ loops for samples Co$\mathrm{SiO}_{x} \mathrm{~N}_{\mathrm{y}}$ and Co-CNT at $\mathrm{T} 1 / 42 \mathrm{~K}$ are presented in Fig. 10a and Fig. 10b, respectively. Data for the magnetization are normalized to the saturation value M s. Similar results are displayed in SI-6 for $\mathrm{T} 1 / 4350 \mathrm{~K}$. Let us recall that the parallel and perpendicular magnetic field orientations were determined relative to the plane of the substrate. For sample Co-SiO $\mathrm{N}_{y}$ in parallel field the magnetization saturates very fast, the saturation field is close to $3 \mathrm{kOe}$. On the contrary, in the perpendicular field, the saturation field is much greater and exceeds $20 \mathrm{kOe}$. The coercivity $\mathrm{H}_{\mathrm{c}}$ and the squareness $M_{r} / M_{s}$ in the parallel field is equal to 820 Oe and 0.56 , correspondingly. In the perpendicular field these quantities quote to $414 \mathrm{Oe}$ and 0.092 , respectively. Rapid saturation in the parallel field along with greater values of the squareness and the coercivity can be reasonably attributed to an easy axis of magnetization which is oriented parallel to the surface of the substrate. This can be naturally explained by the strong DDI between NPs.

Reversely, for Co-CNT sample $\mathrm{M}(\mathrm{H})$ loops approach each other for a parallel and perpendicular field directions (Fig. 10b). In the parallel field, i.e. perpendicular to the nanotube axis, a slightly faster saturation is observed, akin to the magnetization along the easy axis. In the direction of the magnetic field perpendicular to the substrate, i.e. parallel to the nanocylinder axis, the approaching saturation is slightly slower, but the magnetization curve is much closer to the case of the parallel field, compared to, for example, the magnetization of Co nanowires along their easy axes [30]. In Ref. 36 curves of approaching magnetic saturation along easy and hard axes, as well as coercivity and anisotropy constants differ significantly.

Thus, an essential difference in the shape of the magnetization curves is observed when nanosized Co is formed on silicon oxynitride and inside CNT, respectively. In the former case the easy axis of magnetization can be easily identified as oriented in-plane, while in the latter case axes of the Co nanocylinders are neither easy nor hard axes in a first approach. The result obtained for CoCNT sample is not typical for systems of Co nanocylinders (nanowires). Usually in such systems, with aspect ratios equal to 3 and greater, the shape anisotropy dominates, and easy axis is oriented along the cylinder axis and the $\mathrm{M}(\mathrm{H})$ shapes for easy and hard axes directions are very different [36e39]. In our samples the experimental data reveal that this is not the case. Although $\mathrm{M}(\mathrm{H})$ curves slightly differ, they are much closer to each other than usually reported in literature [36e39]. Therefore, most likely, here is a complex case where magnetocrystalline and, possibly, magnetoelastic anisotropy of nanoparticles have a dispersion relative to some direction at a certain angle to the axis of the nanocylinders. 

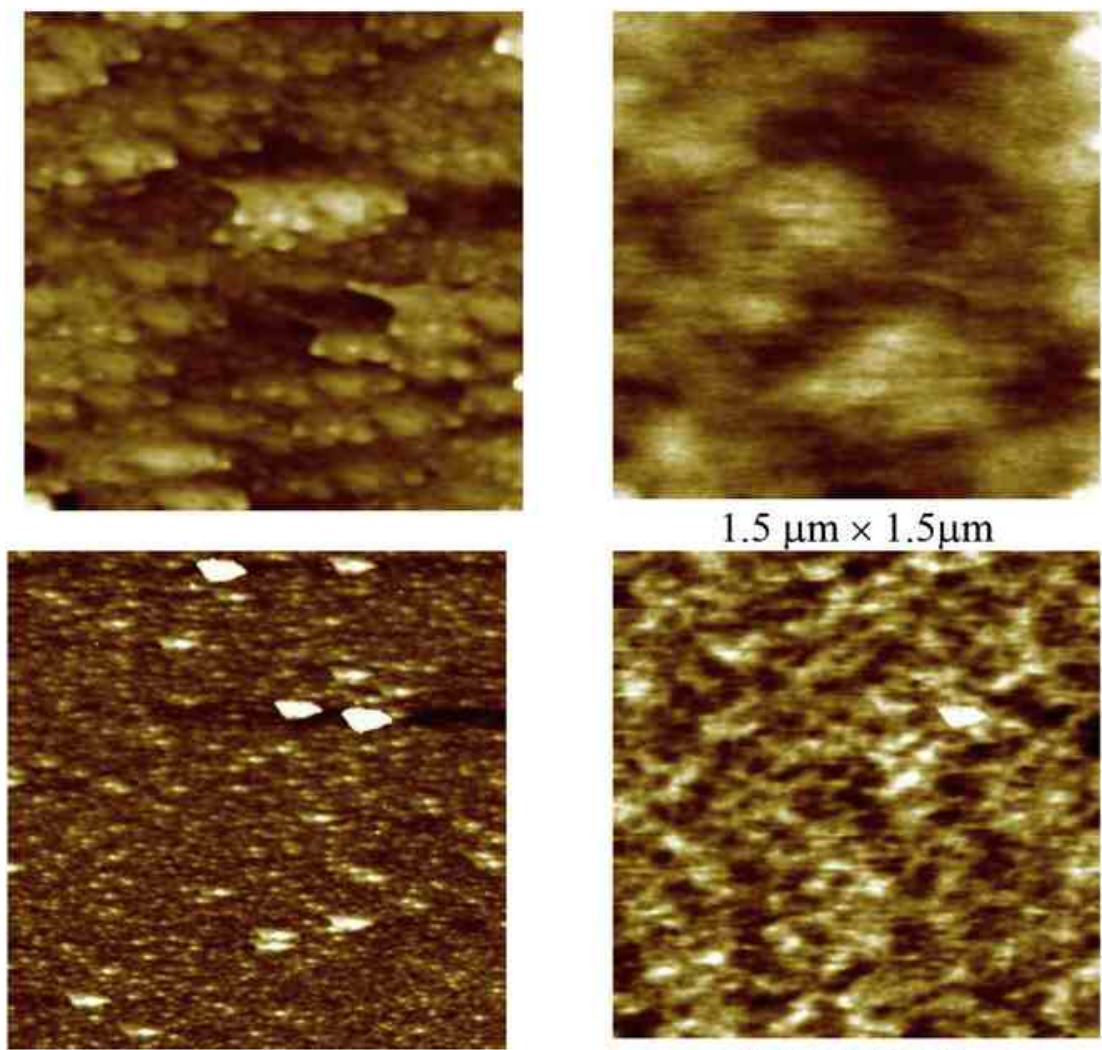

$5 \mu \mathrm{m} \times 8 \mu \mathrm{m}$

Fig. 7. AFM (left) and MFM (right) images of arrays of Co NPs on the top of silicon oxynitride nanocones at different magnifications. version of this figure can be viewed online.)
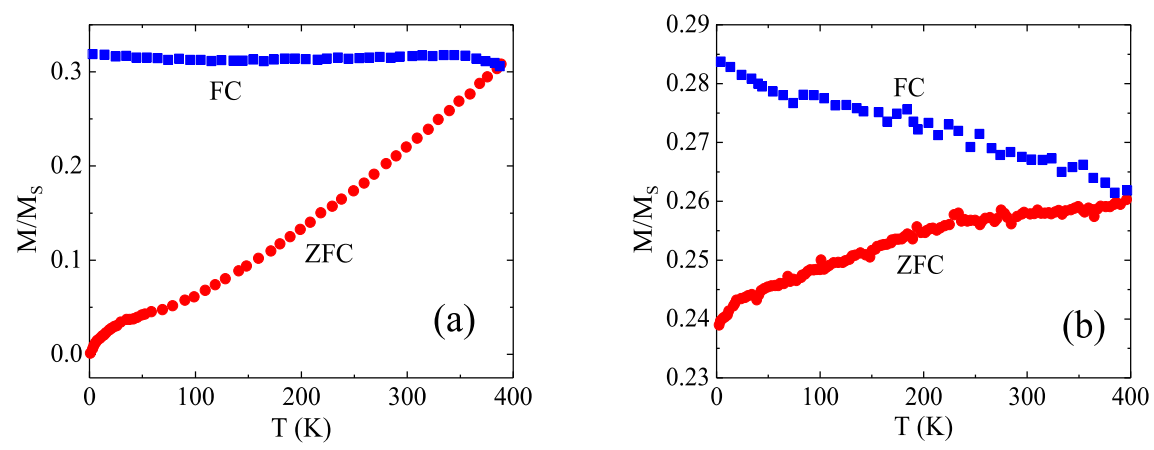

Fig. 8. FC-ZFC magnetizations for (a) Co NPs on the top of silicon oxynitride nanocones; (b) Array of Co NPs embedded inside CNTs. Magnetization was measured at $\mathrm{H} 1 / 425$ Oe. (A colour version of this figure can be viewed online.)

From these results it follows that, the hexagonal c axis of hcp Co does not correlate with the nanocylinder axis as is usually observed for ensemble of Co nanowires [39]. Therefore, the magnetocrystalline anisotropy is neither oriented along the nanocylinder axis nor perpendicular to it. Both the magnetocrystalline anisotropy of the material and the shape anisotropy of nanoparticles embedded inside CNT have a competing effect on the magnetic properties of Co-CNT nanocomposite in this case. The tilt of $c$ axis of hcp Co could be due to the peculiar mechanism of nanosize Co fabrication. Indeed, the formation of an array of cobalt nanoparticles occurs by depositing first a nanosized film on amorphous silicon oxide substrate. At the stage of nucleation, the Co film deposited on a non-oriented substrate does not wet the surface and Co crystallites aggregate with random crystalline orientations
$[40,41]$. The insertion of the NPs inside carbon nanotubes could create stresses and deformation that, in turn, could be the reason of the occurrence of a magnetoelastic anisotropy.

\section{Discussion}

\subsection{Evaluation of the micromagnetic parameters}

In this Section we will evaluate effective micromagnetic parameters like anisotropy $\left(\begin{array}{ll}H & \text { ra }\end{array}\right)$ and exchange $\left(H_{\text {ex }}\right)$ fields, and the effective magnetic anisotropy constant $(\mathrm{K})$. All these quantities are evaluated within the random anisotropy model (RAM) [42e44]. The RAM has been successfully applied in the past to explain the properties of amorphous and nanocrystalline ferromagnets $[44,45]$, 

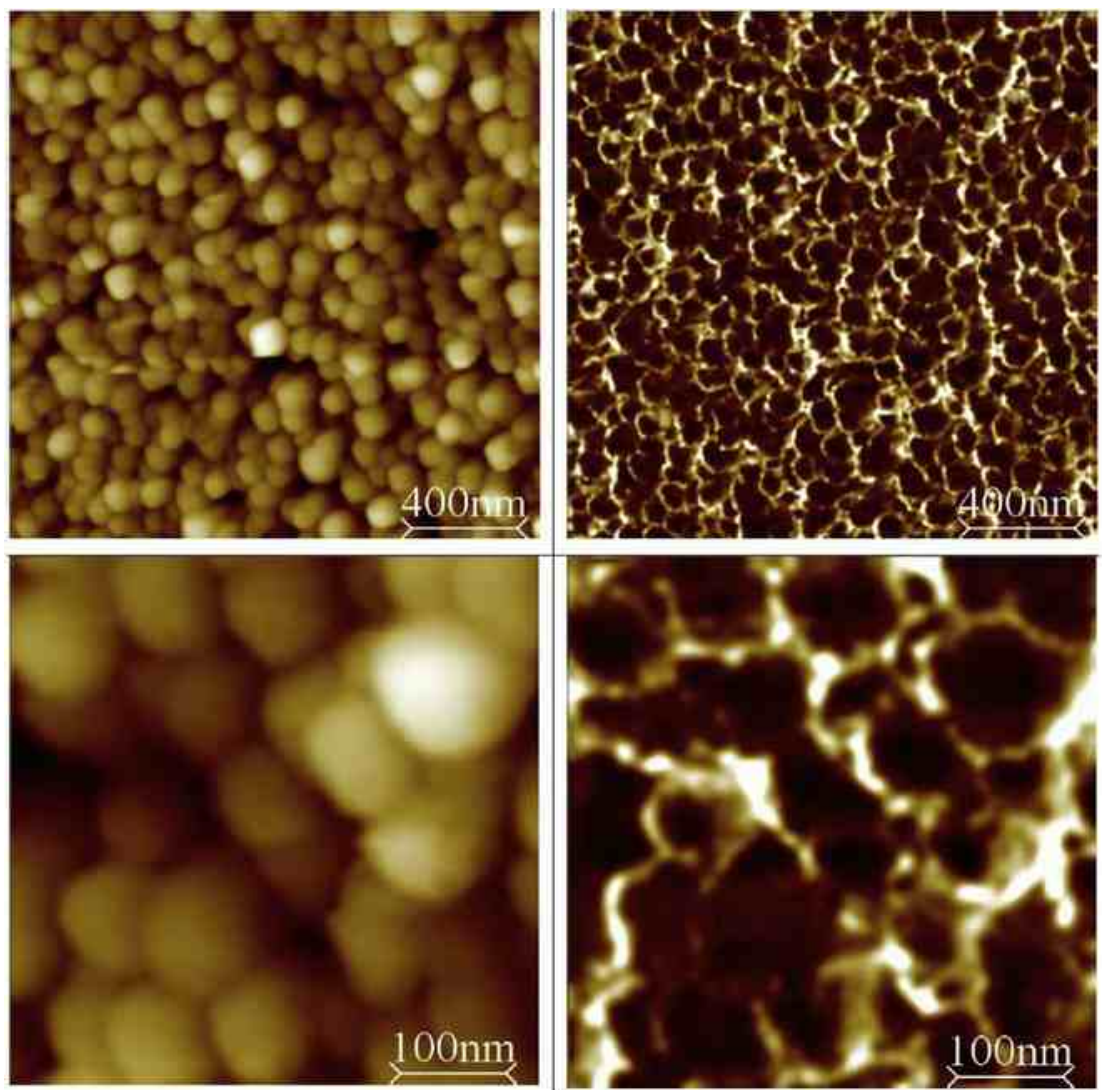

Fig. 9. AFM (left) and MFM (right) images of arrays of Co NPs embedded inside CNTs at different magnifications.

(A colour version of this figure can be viewed online.)
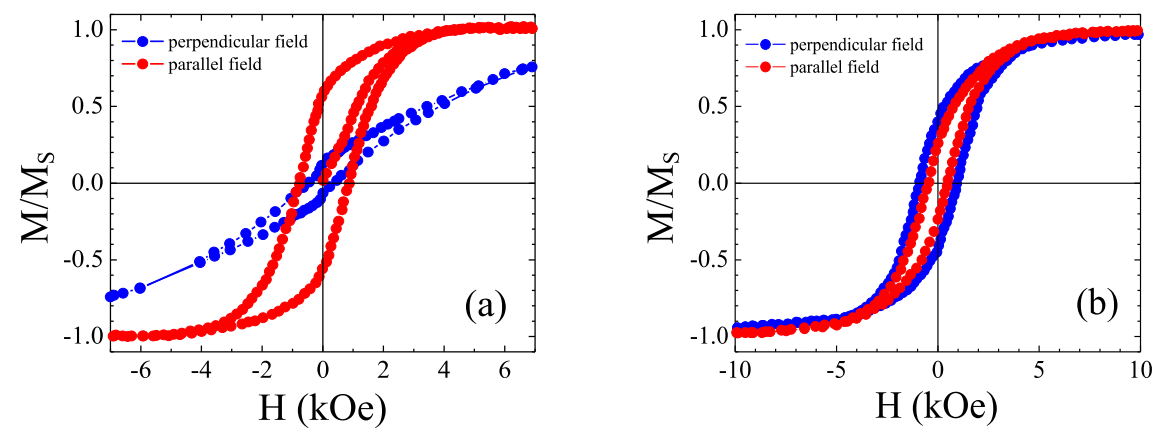

Fig. 10. $M(\mathrm{H})$ curves for the parallel (red color) and perpendicular (blue color) magnetic field orientations. this figure can be viewed online.)

and more recently to explain the properties of ferromagnetic NPs in CNT $[21 \mathrm{e} 24,46,47]$.

The basic equations of the RAM are reduced to the following simple expressions for the micromagnetic parameters $[21,24,43]$.

$\mathrm{H}_{\mathrm{ra}} 1 / 42 \mathrm{~K}=\mathrm{M}_{\mathrm{S}}$;

$H_{e x} 1 / 42 A=M_{S} R_{a}^{2}$

$\mathrm{K} 1 / 4 \mathrm{H}_{C} \mathrm{~A}^{3} \mathrm{M}_{\mathrm{S}}^{. h} 114 \partial \mathrm{k}_{\mathrm{B}} \mathrm{T}_{\mathrm{B}} \mathrm{P}^{\mathrm{i}}$;

where $A$ is the exchange constant, $k_{B}$ is Boltzmann constant, and $R_{a}$ is the length over which the magnetic anisotropy axes are correlated. Usually in a nanocrystalline material $R_{a}$ is assumed to be equal to the radius of the nanoparticle $[21,44]$.

From Eqs. (1)e(3), it follows that in order to evaluate the micromagnetic parameters it is necessary to measure a number of quantities such as $M_{S}, T_{B}, H_{C}, R_{g}$ and to know the exchange constant $A$. The $T_{B}, H_{C}$, and $R_{a}$ were obtained by us directly from the experiments. The $M_{S}$ could also be obtained from the experiment if the exact weight of $\mathrm{Co}$ is known and under the assumption that all the cobalt NPs are ferromagnetic. The weight of Co was estimated based on the average density of the nanoparticle arrangement and their average size. The obtained value was consistent with the amount of evaporated Co according to Table 1. Nevertheless, some uncontrollable distribution of quantities used in such calculations can lead to significant inaccuracies in the $M s$ value. For this reason, we also analyzed the literature data for $\mathrm{M}$ s in nanosized Co samples. The $A$ values were also taken from literature data for the ensemble 
of Co NPs.

It is known that the saturation magnetization for nanosized Co is characterized by significant dependencies on the temperature and size of the NPs $[48,49]$. In the low-temperature region its value for clusters containing more than $550 \mathrm{e} 600$ atoms approximates the value of a bulk material [50], for which M s $1 / 41440 \mathrm{emu} / \mathrm{cm}^{3}$ [51]. Ross et al. assumed that $M_{S} 1 / 41100 \mathrm{emu} / \mathrm{cm}^{3}$ for massive of Co NPs [52]. To calculate the magnetization distribution in Co NPs, it is usually assumed that the saturation magnetization of Co NPs is $M_{S} 1 / 41400 \mathrm{emu} / \mathrm{cm}^{3}$, the exchange constant is given by A $1 / 41.310^{6} \mathrm{erg} / \mathrm{cm}$ and the uniaxial anisotropy constant is $\mathrm{K} 1 / 44.310^{6} \mathrm{erg} / \mathrm{cm}^{3}[53,54]$. As for the exchange constant $A$, it depends less on temperature, but for nanoscale layers or nanoparticle arrays its value may depend on the method by which it is measured. Measurements of $A$ indicate a large discrepancy in the values: $1.8 \pm 0.310^{11} \mathrm{~J} / \mathrm{m}$ from Brillouin light scattering (BLS) [55], $2.7 \pm 0.110^{11} \mathrm{~J} / \mathrm{m}$ from neutron scattering [56], and $2.1 \quad 10^{11} \mathrm{~J} / \mathrm{m}$ from ferromagnetic resonance studies [57]. It was established in Ref. [58] from BLS data that $A$ and $M s$ of the Co layer of $80 \mathrm{~nm}$ are equal to $2.110^{11} \mathrm{~J} / \mathrm{m}$ and $1332 \mathrm{emu} / \mathrm{cm}^{3}$, respectively, and for $10 \mathrm{~nm}$ thickness $A$ was found to be $1.54 \pm 0.1210^{11} \mathrm{~J} / \mathrm{m}$ and $M_{S}$ was $1247 \pm 44 \mathrm{emu} / \mathrm{cm}^{3}$.

Based on the experimental data and analysis of the literature, we evaluated $\mathrm{H}_{\mathrm{ra}}$ and $\mathrm{H}_{\mathrm{ex}}$ values together with $\mathrm{K}$. All measured and evaluated data are summarized in Table 5 for Co-CNT and Co$\mathrm{SiO}_{\mathrm{X}} \mathrm{N}_{\mathrm{y}}$ systems.

It follows from the analysis for both samples that the exchange field is less than the anisotropy field, which could indicate the negligible role of the interparticle exchange coupling. It is also confirmed by relatively high values of the anisotropy constant, (2.65e3.77) $10{ }^{5} \mathrm{~J} / \mathrm{m}^{3}$ and random anisotropy field, (3.76e5.35) kOe, which could be due to shape, magnetocrystalline and magnetoelastic anisotropies. In the above considerations the DDI was absent. Obviously, for densely packed NPs its contribution could be essential. These arguments require further analysis.

\subsection{Comparative analysis of the contributions of SA and DDI}

The total anisotropy energy is determined by the contributions of the magnetocrystalline ( $\mathrm{K} \quad \mathrm{MCA}$ ) and shape anisotropy ( $\left.\begin{array}{l}\mathrm{K} \\ \mathrm{SA}\end{array}\right)$, the magnetoelastic contribution ( $K$ MEA), the DDI $\left(K_{D D}\right)$ and possibly the surface anisotropy. We will neglect this last term further in this study. The effective anisotropy constant $\mathrm{K}$ found above, determines the total contribution of all components $[60,61]$.

$$
K_{1 / 4} K_{M C A} p K_{S A} p K_{\text {DD }} p K_{\text {MEA }}
$$

The shape anisotropy is determined by the interaction of the particle with its own demagnetizing field. For a homogeneous magnetization the corresponding SA constant is [54].
$\mathrm{K}_{\mathrm{SA}} 1 / 4 \mathrm{~d} 1=2 \mathrm{mb} \mathrm{M}_{\mathrm{S}}^{2} \mathrm{DN}$

where DN $1 / 4 N_{k}-N_{\perp}, N_{k}$ is the demagnetizing factor of the NP along the symmetry axis $\mathrm{z}$ and $\mathrm{N}_{\perp}$ is the demagnetizing factor in the direction perpendicular to $z$ axis.

The contribution of the DDI between NPs is expressed as $[62,63]$.

$K_{D D} 1 / 4$ ठ1=8pp $m_{0} M_{S}^{2} V^{*} z^{3} s_{d}$

where $\mathbf{Z}$ is an average interparticle distance, $\mathbf{S}_{d}$ is a lattice sum that depends on the type of lattice in the NPs arrangement, their shape, on the dimensionality of the sample and on the mutual orientation of the NPs magnetizations, $V$ is the NP volume.

The contribution of the SA for the sample Co-SiO ${ }_{x} \mathrm{~N}_{y}$ can be neglected due to the almost equal transverse and longitudinal demagnetizing factors $(\mathrm{DN}<<\mathrm{P}), \mathrm{P}$ being the volume fraction occupied by the ferromagnetic material. Due to the spherical shape of NPs in this case it seems reasonable to also neglect the possible contribution of the magnetoelastic anisotropy. Therefore, the anisotropy constant $\mathrm{K}$ in this case is a function of only $\mathrm{K}_{\mathrm{DD}}$ and $\mathrm{K}_{\mathrm{MCA}}$. To estimate the contribution of the DDI, we follow Eq. (6), in which for two-dimensional arrays of NPs the value of the lattice sum $S$ d $1 / 4$ $6 e 8$ was applied $[64,65]$.

For the arrays of Co-CNT, we cannot neglect the possible contribution of SA, MCA and MEA. For these samples the total anisotropy constant will be determined by the expression (4). The contribution of the SA and DDI of the system of magnetic nanocylinders is expressed as [66].

$\mathrm{K}_{\mathrm{SA}} \mathrm{p} \mathrm{K}_{\mathrm{DD}} 1 / 4$ ठ1 =4Fm $\mathrm{M}_{\mathrm{S}}^{2} \circlearrowright 13 \mathrm{PP} ;$

here $\mathrm{P} 1 / 4\left(p r^{2} S\right)$ is a volume fraction occupied by ferromagnetic nanocylinders, $r$ is radius of the nanocylinder, $S$ is surface density of the nanocylinders. In Table 6 we present the results of the evaluations of the SA, MCA and DDI for both samples for the parallel field. We do not show $\mathrm{K}_{\text {MEA }}$ in this Table. The discussion of this issue will be done in the next Section.

Results presented in Table 6 reveal that in the case of $\mathrm{Co}_{\mathrm{SiN}} \mathrm{O}_{\mathrm{y}}$ array, in which the NP shape is close to spherical, $\mathrm{K}_{\mathrm{MCA}}$ is slightly greater that $K_{D D}$. Nevertheless, the impact of the DDI in the presence of only MCA is determined by the dipolar coupling constant a $1 / 4 K_{D D}=K_{M C A}$ [67]. There is a crucial parameter $a_{C r}$ that determines the transition from single-particle to collective behavior. The reported values for fcc or hcp Co NPs are $a_{c r} 1 / 4(0.2 e 0.4)(d / z)^{3}$ [68]. If $\mathrm{a}>\mathrm{a}_{\mathrm{cr}}$, then DDI dominates, otherwise the MCA prevails. For our geometry we get $a_{c r} z 0.14<a 1 / 40.61$, which indicates that the contribution of the DDI is sufficient for the formation of regions covering many NPs, that is indeed observed experimentally.

For the Co-CNT array we have to consider both the SA and MEA.

Table 5

Micromagnetic parameters of Co-CNT and Co-SiO ${ }_{x} \mathrm{~N}_{y}$ systems as obtained within RAM.

\begin{tabular}{|c|c|c|c|c|c|c|}
\hline \multirow[t]{2}{*}{ Parameter } & \multirow[t]{2}{*}{ Unit } & \multicolumn{2}{|l|}{ Parallel field } & \multicolumn{2}{|c|}{ Perpendicular field } & \multirow[t]{2}{*}{ Reference note } \\
\hline & & Co-CNT & $\mathrm{Co}-\mathrm{SiO}_{x} \mathrm{~N}_{\mathrm{y}}$ & Co-CNT & Co-SiO $N_{y}$ & \\
\hline $\mathrm{H}_{\mathrm{c}}$ & $\mathrm{Oe}$ & 473 & 820 & 1050 & & Experiment \\
\hline $\mathrm{M}_{\mathrm{S}}$ & $\mathrm{A} / \mathrm{m}$ & $1.410^{6}$ & $1.410^{6}$ & $1.410^{6}$ & $1.410^{6}$ & Experiment and Ref. $[53,54]$ \\
\hline$A$ & $\mathrm{~J} / \mathrm{m}$ & $1.54 \quad 10 \quad 11$ & $1.5410^{11}$ & $1.54 \quad 10^{11}$ & $1.5410^{11}$ & Ref. [59] \\
\hline $\mathrm{T}_{\mathrm{B}}$ & $\mathrm{K}$ & 400 & 400 & $\sim 500$ & 314 & Experiment \\
\hline $\mathrm{M}_{\mathrm{r}} / \mathrm{M}_{\mathrm{S}}$ & & 0.25 & 0.25 & 0.45 & 0.45 & Experiment \\
\hline $\mathrm{K}$ & $\mathrm{J} / \mathrm{m}^{3}$ & $2.6510^{5}$ & $3.7710^{5}$ & $3.1510^{5}$ & $3.1510^{5}$ & Calculations \\
\hline $\mathrm{H}_{\mathrm{ra}}$ & $\mathrm{kOe}$ & 3.76 & 5.35 & 4.48 & 4.48 & Calculations \\
\hline $\mathrm{H}_{\mathrm{ex}}$ & $\mathrm{kOe}$ & 1.4 & 2.19 & 1.4 & 2.19 & Calculations \\
\hline
\end{tabular}


Table 6

Evaluations of the SA, MCA and DDI for the parallel orientation of the magnetic field $d$ is an average diameter of the NP and $L$ is an average height of the nanocylinder

\begin{tabular}{|c|c|c|c|}
\hline Parameter & Unit & $\mathrm{Co}-\mathrm{SiO}_{x} \mathrm{~N}_{\mathrm{y}}$ & Co-CNT \\
\hline $\mathrm{K}_{\mathrm{SA}}$ & $\mathrm{J} / \mathrm{m}^{3}$ & $\mathrm{e}$ & $6.16 \quad 10 \quad 5$ \\
\hline$K_{D D}$ & $\mathrm{~J} / \mathrm{m}^{3}$ & $1.410^{5}$ & $1.0910^{5}$ \\
\hline $\mathrm{K}_{\mathrm{MCA}}$ & $\mathrm{J} / \mathrm{m}^{3}$ & $2.3710^{5}$ & $2.3710^{5}$ \\
\hline $\mathrm{K}_{\mathrm{SA}} \mathrm{bK} \mathrm{K}_{\mathrm{DD}}$ & $\mathrm{J} / \mathrm{m}^{3}$ & $\mathrm{e}$ & $\begin{array}{lll}5.07 & 10 & 5\end{array}$ \\
\hline a $1 / 4 \mathrm{~K}_{\mathrm{DD}} / \mathrm{K}_{\mathrm{MCA}}$ & a.u. & 0.61 & $\mathrm{e}$ \\
\hline$z$ & $\mathrm{~nm}$ & 32.6 & 51.5 \\
\hline S & $\mathrm{cm}^{2}$ & 31010 & 1.21010 \\
\hline$P$ & a.u. & 0.15 & 0.06 \\
\hline d & $\mathrm{nm}$ & $25 \pm 8$ & $15 \pm 5$ \\
\hline L & $\mathrm{nm}$ & e & $100 \mathrm{e} 150$ \\
\hline
\end{tabular}

Their contribution along with the MCA should exceed the contribution of the dipole interaction between NPs.

\subsection{Contribution of the magnetoelastic anisotropy (MEA)}

Let us estimate the possible contribution of elastic stresses to the energy of magnetic anisotropy in Co-CNT samples. The total anisotropy energy could be determined by Eq.

(4) $[60,61]$. All constants in Eq. (4) except the magnetoelastic anisotropy, have been already determined and given in Table 6 . We reasonably assume that the $\mathrm{K}_{\text {MCA }}$ evaluated for the $\mathrm{Co}-\mathrm{SiO}_{\mathrm{x}} \mathrm{N}_{\mathrm{y}}$ samples will be the same as for Co-CNT. On the base of these data we obtained $\mathrm{K}_{\mathrm{MEA}} \mathrm{Z}$ $5.3510^{5} \mathrm{~J} / \mathrm{m}^{3}$.

It should be noted that the type of crystalline lattice of Co affects significantly the interpretation of data on the magnetoelastic anisotropy [69]. For fcc Co the constant of the magnetoelastic anisotropy can be evaluated as

$\mathrm{K}_{\mathrm{MEA}}^{\mathrm{fcc}} \mathrm{z}$ ð3 $=2 \mathrm{HS}$;

where $\mathrm{I}$ is the magnetostriction constant and $\mathrm{S}$ is the elastic stress [70]. Using for fcc Col 1/450 $10 \quad{ }^{6}$ [71] and applying the obtained $\mathrm{K}_{\mathrm{MEA}}$ value we get $\mathrm{S}$ order of $7.13 \mathrm{GPa}$.

For hcp Co when the hexagonal axis [0001] is parallel to the CNT axis and neglecting the torsional stresses, the constant of the magnetoelastic anisotropy can be expressed as [72].

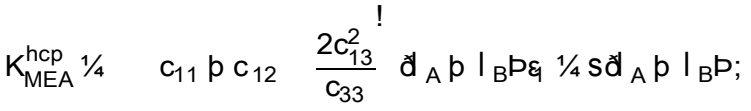

where $c_{i j}$ are elastic stiffness constants, the indices $i, j 1 / 41,2,3$ number the axes of the hexagonal crystal (here indices 3 corresponds to the hexagonal axis [0001]), $\mathrm{I}_{\mathrm{A}}$ and $\mathrm{I}_{\mathrm{B}}$ are magnetostriction constants and $\varepsilon_{1}$ is strain. Substituting in Eq. (9) $\mathrm{I}_{\mathrm{A} 1 / 4} 5010^{6}, \quad \mathrm{I}_{\mathrm{B}} 1 / 410710 \quad 6 \quad$ [73], $\mathrm{C}_{11} 1 / 4307.1 \mathrm{GPa}$; $\mathrm{C}_{12} \frac{1}{4} 165.0 \mathrm{GPa} ; \mathbb{\Phi}_{3} 1 / 4102.7 \mathrm{GPa} ; \S_{3} 1 / 4358.1 \mathrm{GPa}$ [74] and using the previously obtained K hcp $1 / 45.3510^{5} \mathrm{~J} / \mathrm{m}^{3}$, we get $\varepsilon_{1} 1 / 40.00825$ which leads to the tensile stresses $\mathrm{s} z 3.408 \mathrm{GPa}$.

For the hexagonal axis 3 oriented perpendicular to the CNT axis the exact expression for the $\mathrm{K}$ MEA depends on the orientation of crystal magnetization $\mathrm{m}$ [69],

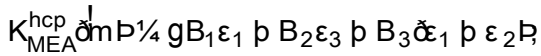

where $g \frac{1}{4} \quad a_{1}^{2} p \frac{\varepsilon_{2}}{\varepsilon_{1}} a_{2}^{2} \quad \approx a_{1}^{2} p \quad a_{2}^{2} p$ and $a_{i}$ are the direction cosines of the magnetization with respect to the hexagonal axes, $\mathrm{B}_{\mathrm{i}}$ are magnetoelastic coupling coefficients. In these calculations we used
$B_{1} 1 / 48.110{ }^{6} \mathrm{~J} / \mathrm{m}^{3}, B_{2} 1 / 42910{ }^{6} \mathrm{~J} / \mathrm{m}^{3}, B_{3} 1 / 428.210^{6} \mathrm{~J} / \mathrm{m}^{3}[69]$ The strain $\varepsilon$ is found from the system of equations.

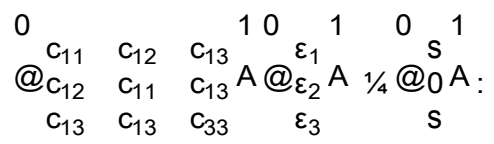

If axis 1 is oriented perpendicular and axis 2 parallel to the CNT axis, then we get from Eq. (11) $\varepsilon_{1} 1 / 40.00403 \mathrm{~s}, \varepsilon_{2} \frac{1}{4} 0.00300 \mathrm{~s}$, $\varepsilon_{3} \frac{1 / 4}{4} 0.00250 \mathrm{~S}$, where $S$ is in GPa. And for limiting cases $a_{1} 1 / 40$ and $a_{2} 1 / 40$ the value of $g$ varies within the range $3 / 4 \ldots 1$. This leads to boundary values of stresses from $S 1 / 45.42 \mathrm{GPa}$ to $S 1 / 421.93 \mathrm{GPa}$. The real value of stresses belongs to this interval. A similar result is obtained when the axis 3 and the axis 2 are perpendicular and the axis 1 is parallel to the axis of the CNT. However, the exact values of the direction cosines $a_{i}$ are not known in our case. Therefore, for an approximate estimation of $\mathrm{K}$ hcp we assume that the first term in Eq. (10) can be neglected and Eq. (10) can be rewritten as $K{ }_{M E A}^{h c p} \partial^{\prime} 1 / 4$ $B_{2} \varepsilon_{3} p B_{3} \partial \varepsilon_{1} p \varepsilon_{2} P$. In this case the value of stress iss $1 / 49.515 \mathrm{GPa}$. This is valid for both cases, when the axis 3 is perpendicular to the axis of the CNT, and the axis 1 is either perpendicular or parallel to the axis of the CNT.

The elastic stiffness constants $c_{i j}$, generally speaking, depend on the stress $S$. This dependence is close to linear, $c_{i j}(S) 1 / 4(1 \mathrm{p} \mathrm{k} \mathrm{S}) c_{i j 0}$, where $\mathrm{C}_{\mathrm{ij} 0}$ are the table values for stresses close to zero and k z $0.017 \mathrm{GPa}^{1}$ is an empirical coefficient $[75,76]$. Thus, considering the dependence of the magnetoelastic constants on the compression value, we have S $1 / 411.351 \mathrm{GPa}$. Such internal elastic stresses could cause the deformation of the CNT lattice only in the range of $0.5 \mathrm{e} 2 \%$, which is in good agreement with the literature [77].

The obtained $\mathrm{K}_{\mathrm{MEA}}$ values reveal that the largest contribution of the magnetoelastic anisotropy occurs in the direction parallel to the substrate and when the hexagonal [0001] axes is oriented along the field direction. Therefore, we may conclude that the DDI for Co-CNT samples is overcome by the shape and magnetoelastic anisotropies. In the latter case the contribution of the hcp Co dominates. The $c$ axes of the hcp phase with high probability is oriented perpendicular to the CNT axis. This assumption has been confirmed by the results of micromagnetic simulation, which was performed for hcp Co nanocylinders with diameter $20 \mathrm{~nm}$ and length $100 \mathrm{~nm}$. We applied the Nmag package [78] based on the Landau-LifshitzGilbert equation and finite elements method. The discretization length was chosen to be $1.5 \mathrm{~nm}$ that should be enough for the acceptable accuracy because the exchange length of cobalt is about $10 \mathrm{~nm}$ [79]. Axis $Z$ is oriented along the nanocylinder axis (i.e., the CNT orientation), and $X Y$ plane is lying in the direction perpendicular to the CNT axis. Moreover, the $X$ axis coincides with the [0001] axis of the hcp Co.

The simulation shows that in such conditions the relaxed magnetization configuration of the cylinder depends on the $\mathrm{K}$ MEA (see Fig. 11). For the absence of the magnetoelastic stress, the magnetization of the Co nanocylinder is homogeneous and oriented along the $Z$ axis, see Fig. 11a. The presence of the magnetoelastic stresses, $\mathrm{K}_{\mathrm{MEA}} 1 / 45.3510^{5} \mathrm{~J} / \mathrm{m}^{3}$, breaks nanocylinder on two domains, Fig. 11b. The orientation of the magnetization within the domain is oriented along the $X$ axis. The performed simulations of the magnetic structure of Co nanocylinders should be considered only as evaluation results showing that with the magnetoelastic component nanocylinders may be partitioned into domains, which leads to a decrease in remanence. This correspond well to low remanence in Co-CNT samples. For hcp Co with c axis oriented along the axis of CNT, the reasonable values of $\mathrm{K}_{\mathrm{MEA}}^{\text {hcp }}$ do not create the multidomain structure (this result is not shown here). 


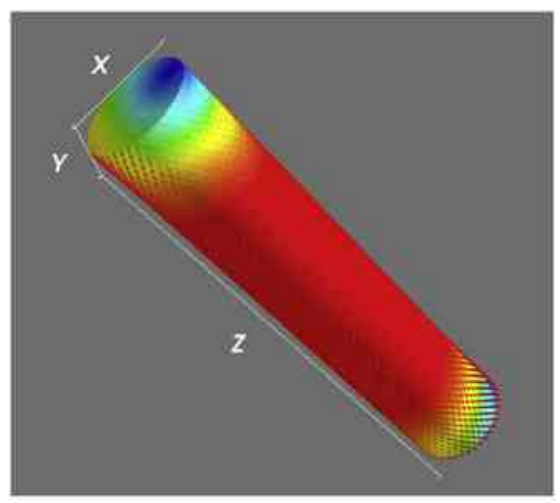

a)

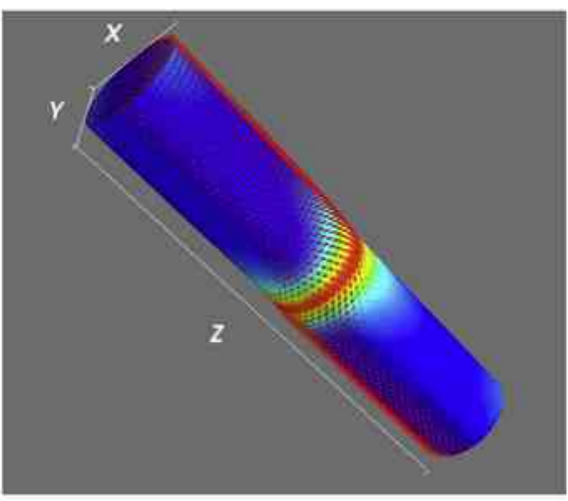

b)

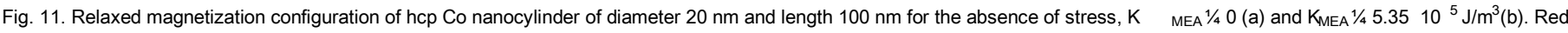
color corresponds to the magnetization direction along the nanocylinder axis, blue color corresponds to the magnetization direction oriented perpendicular to the nanocylinder axis. (A colour version of this figure can be viewed online.)

\section{Conclusion}

The analysis of the magnetic properties of nanosized Co on the top of $\mathrm{SiO}_{x} \mathrm{~N}_{\mathrm{y}}$ nanocones and inside CNT has been performed. The average density of $\mathrm{Co}$ inclusions is similar for both type of samples and is order of $10{ }^{10} \mathrm{~cm}^{2}$. Nevertheless, these two systems behave differently in a magnetic field. In the former case the Co NPs are strongly coupled via the DDI. As a result, the easy axis plane is oriented parallel to the substrate. For Co-CNT samples Co nanoparticles are magnetically isolated. The reason for a minor effect of the DDI is the magnetic anisotropy. It increases significantly because of the peculiar morphology of Co inclusions in CNT and stresses caused by the carbon nanotubes. We evaluated the values of shape, magntocrystalline and magnetoelastic anisotropy constants. The magnetoelastic anisotropy has been evaluated for both observed crystalline structures of Co, fcc and hcp. It has been demonstrated that due to the presence of the hcp crystalline phase, stresses could reach significant values leading to noticeable magnetoelasticity. The influence of stresses onto magnetic structure of Co inclusions has been investigated by the micromagnetic simulations, which helped us to determine the probable direction of the hexagonal axis oriented perpendicular to the CNT axis. The developed approach in the fabrication of an ensemble of anisotropic nanoparticles can be very useful in the design of carbon-based magnetoelectronic devices.

\section{Acknowledgements}

$\mathrm{P}$. Legagneux (Thales) is acknowledged for purchasing $\mathrm{SiO}{ }_{2} / \mathrm{Si}$ substrate by electron cyclotron resonance plasma process; Derory (IPCMS/GMO), J. Faerber (IPCMS/GSI) are acknowledged for SQUID measurements and SEM observations, respectively. C. Meny (IPCMS/GEMM) is acknowledged for helpful discussions. ALD and SLP acknowledge the financial support from the Belaruisian State Program "Photonics, opto- and microelectronics", grant No. 3.3.01.

\section{Appendix A. Supplementary data}

Supplementary data related to this article can be found at https://doi.org/10.1016/j.carbon.2018.08.024.

\section{References}

[1] R.D. Zysler, E. De Biasi, C.A. Ramous, D. Fiorani, H. Romero, Surface and interparticle effects in amorphous magnetic nanoparticles, in: D. Fiorani (Ed.),
Surface Effects in Magnetic Nanoparticles, Springer, 2005, pp. $239 \mathrm{e} 262$.

[2] J.L. Dormann, D. Fiorani, E. Tronc, Magnetic relaxation in fine-particle systems, Adv. Chem. Phys. 98 (1997) 283e494.

[3] W. Luo, S.R.Nagel, T.F.Rosenbaum, R.E.Rosensweig, Dipole interactions with random anisotropy in a frozen ferrofluid, Phys. Rev. Lett. 67 (19) (1991) $2721 \mathrm{e} 2724$.

[4] S. Mørup, M.F. Hansen, C. Frandsen, Magnetic interactions between nanoparticles, Beilstein J. Nanotechnol. 1 (2010) 182e190.

[5] X. Batlle, A. Labarta, Finite-size effects in fine particles: magnetic and transport properties, J.Phys. D 35 (6) (2002) R15eR42.

[6] A.S. Andreev, M.A. Kazakova, A.V. Ishchenko, A.G. Selyutin, O.B. Lapina, V.I. Kuznetsov, et al., Magnetic and dielectric properties of carbon nanotubes with embedded cobalt nanoparticles, Carbon 114 (2017) 39e49.

[7] E. Tronc, A. Ezzir, R. Cherkaoui, C. Chaneac, M. Nogues, H. Kachkachi, et al., Surface-related properties of $\mathrm{g}-\mathrm{Fe}_{2} \mathrm{O}_{3}$ nanoparticles, J. Magn. Magn Mater. 221 (1e2) $(2000) 63 e 79$.

[8] W. Baaziz, S. Begin-Colin, B.P.Pichon, I. Florea, O. Ersen, S. Zafeiratos, et al., High-density monodispersed cobalt nanoparticles filled into multiwalled carbon nanotubes, Chem. Mater. 24 (2012) 1549e1551.

[9] C. Petit, A. Taleb, M.P. Pileni, Self-organization of magnetic nanosized cobalt particles, Adv. Mater. 10 (3) (1998) 259e261.

[10] S.Sun, C.B.Murray, Synthesis of monodisperse cobalt nanocrystals and their assembly into magnetic superlattices, J. Appl. Phys. 85 (8) (1999) 4325e4330.

[11] J.S. Lee, R.P. Tan, J.H. Wu, Y.K. Kim, Effect of interparticle interactions and size dispersion in magnetic nanoparticle assemblies: a static and dynamic study, Appl. Phys. Lett. 99 (6) (2011), 062506-1-3.

[12] Z. Sabsabi, F. Vernay, O. Iglesias, H. Kachkachi, Interplay between surface anisotropy and dipolar interactions in an assembly of nanomagnets, Phys. Rev B 88 (10) (2013), 104424-1-12.

[13] D. Xiao, T. Lu, R. Zeng, Y. Bi, Preparation and highlighted applications of magnetic microparticles and nanoparticles: a review on recent advances, Microchim Acta 183 (10) (2016) 2655e2675.

[14] W. Luo, W.H. Doh, Y.T. Law, F. Aweke, A. Ksiazek-Sobieszek, A. Sobieszek, et al., Single-layer graphene as an effective mediator of the metal-support interaction, J.Phys. Chem. Lett. 5 (11) (2014) 1837e1844.

[15] A. Dolgiy, S. Redko, H. Bandarenka, S.L. Prischepa, K. Yanushkevich, P. Nenzi, et al., Electrochemical deposition and characterization of $\mathrm{Ni}$ in mesoporous silicon, J.Electrochem. Soc. 159 (10) (2012) D623eD627.

[16] G. Xiao, S.H. Liou, A. Levy, J.N.Taylor, C.L.Chien, Magnetic relaxation in Fe$\left(\mathrm{SiO}_{2}\right)$ granular films, Phys. Rev. B 34 (11) (1986) 7573e7577.

[17] J.M. Vargas, W.C. Nunes, L.M. Socolovsky, M. Knobel, D. Zanchet, Effect of dipolar interaction observed in iron-based nanoparticles, Phys. Rev. B 72 (18) (2005), 184428-1-6.

[18] F. Faupel, V. Zaporojtchenko, T. Strunkus, M. Elbahri, Metal-polymer nanocomposites for functional applications, Adv. Eng. Mater. 12 (12) (2010) $1177 \mathrm{e} 1190$.

[19] S.L.Prischepa, A.L. Danilyuk, A.L. Prudnikava, I.V. Komissarov, V.A. Labunov, K.I. Yanushkevich, F. Le Normand, Magnetic properties of nanocomposites based on magnetically functionalized carbon nanotubes, in: J.M. Gonzalez Estevez (Ed.), Nanomagnetism, One Central Press (OCP), Manchester, 2014, pp. $227 \mathrm{e} 245$

[20] V.A. Labunov, A.L. Danilyuk, A.L. Prudnikava, I. Komissarov, B.G Shulitski, C. Speisser, et al., Microwave absorption in nanocomposite material of magnetically functionalized carbon nanotubes, J.Appl. Phys. 112 (2) (2012), 024302-1-9.

[21] A.L. Danilyuk, A.L. Prudnikava, I.V. Komissarov, K.I. Yanushkevich, A. Derory, F. Le Normand, et al., Interplay between exchange interaction and magnetic anisotropy for iron-based nanoparticles in aligned carbon nanotube arrays 
Carbon 68 (3) (2014) 337e345.

[22] S.L.Prischepa, A.L. Danilyuk, A.L. Prudnikava, IV. Komissarov, V.A. Labunov, F. Le Normand, Exchange coupling and magnetic anisotropy for different concentration of iron based nanoparticles in aligned carbon nanotube arrays, Phys. Status Solidi C 11 (5e6) (2014) 1074e1079.

[23] A.L. Danilyuk, I.V. Komissarov, A.V. Kukharev, F. Le Normand, J.M. Hernandez, J. Tejada, S.L. Prischepa, Impact of CNT medium on the interaction between ferromagnetic nanoparticles, Europhys. Lett. 117 (2) (2017), 27007-1-7.

[24] A.L. Danilyuk, I.V. Komissarov, V.A. Labunov, F. Le Normand, A. Derory, J.M. Hernandez, et al., Manifestation of coherent magnetic anisotropy in a carbon nanotube matrix with low ferromagnetic nanoparticle content, N. J. Phys. 17 (2) (2015), 023073-1-12.

[25] E.M. Chudnovsky, R.A. Serota, Phenomenological theory of amorphous magnets with small random anisotropy, J Phys C 16 (21) (1983) 4181e4190.

[26] J. Klinovaja, D. Loss, RKKY interaction in carbon nanotubes and graphene nanoribbons, Phys. Rev. B 87 (4) (2013), 045422-1-11.

[27] C.S.Cojocaru, F. Le Normand, On the activation mode in the plasma- and hot filaments-enhanced catalytic chemical vapor deposition of vertically aligned carbon nanotubes, Thin Solid Films 515 (2006) 53e58.

[28] J. Mane Mane, C.S. Cojocaru, A. Barbier, J.P. Deville, B.T. Sendja, F. Le Normand GISAXS study of the alignment of oriented carbon nanotubes grown on plain $\mathrm{SiO}_{2} / \mathrm{Si}(100)$ substrates by a catalytically enhanced CVD process, Phys. Status Solidi 204 (12) (2007) 4209e4229.

[29] S.M. Castanho, R. Moreno, J.L.G.Fierro, Surface oxidation of SiNx green compacts: effect of sintering aids, J.Eur. Ceram. Soc. 17 (1997) 383e391.

[30] S. Doniach, M. Sunjic, Many-electron singularity in X-ray photoemission and X-ray line spectra from metals, J Phys C 3 (1970) 285e291.

[31] L.E. Klebanoff, Spin-resolved x-ray photoemission studies of ferromagnetic metals, J.Vac. Sci. Technol. B14 (1996) 3140e3147.

[32] C.S.Cojocaru, A. Senger, F. Le Normand, A nucleation and growth model of vertically oriented carbon nanofibers or nanotubes by plasma-enhanced catalytic chemical vapor deposition, J.Nanosci. Nanotechnol. 6 (2006) 1 e8.

[33] J. Mane Mane, F. Le Normand, R.E. Medjo, C.S. Cojocaru, O. Ersen, A. Senger, et al., Alignment of vertically aligned carbon nanostructures studied by X-ray absorption spectroscopy, Mater. Sci. Appl. 5 (13) (2014) 966 e983.

[34] E. Tronc, P. Prene, J.P.Jolivet, F. d'Orazio, F. Lucari, D. Fiorani, et al., Magnetic behavior of $\mathrm{g}-\mathrm{Fe}_{2} \mathrm{O}_{3}$ nanoparticles by $\mathrm{M}$ essbauer spectroscopy and magnetic measurements, Hyperfine Interact. 95 (1995) 129 e148.

[35] H. Mamiya, I. Nakatani, T. Furubayashi, Blocking and freezing of magnetic moments for iron nitride fine particle systems, Phys. Rev. Lett. 80 (1) (1998) $177 \mathrm{e} 180$.

[36] K. Gandha, K. Elkins, N. Poudyal, X. Liu, J.P. Liu, High energy product developed from cobalt nanowires, Sci. Rep. 4 (2014), 5345-5.

[37] J.Sanchez-Barriga, M. Lucas, F. Radu, E. Martin, M. Multigner, P. Marin, et al., Interplay between the magnetic anisotropy contributions of cobalt nanowires, Phys. Rev. B 80 (18) (2009), 184424-1-8.

[38] M. Gong, Q. Dai, S. Ren, Magnetic dipolar interaction induced cobalt nanowires, Nanotechnology 27 (7) (2016), 07LT02-1-7.

[39] C. Li, Q. Wu, M. Yue, H. Xu, S. Palaka, K. Elkins, J.P. Liu, Manipulation of morphology and magnetic properties in cobalt nanowires, AIP Adv. 7 (5 (2017), 056229-1-5.

[40] S. Armyanov, Crystallographic structure and magnetic properties of electrodeposited cobalt and cobalt alloys, Electrochim. Acta 45 (2000) 3323e3335.

[41] O. Kitakami, S. Okamoto, Y. Shimada, Effect of surface free energy of underlayer materials on crystal growth of Co polycrystalline films, J.Appl. Phys. 79 (9) (1996) 6880e6883.

[42] R. Harris, M. Plischke, M.J. Zuckerman, New model for amorphous magnetism, Phys. Rev. Lett. 31 (3) (1973) 160e162.

[43] R. Alben, J.J. Becker, M.C. Chi, Random anisotropy in amorphous ferromagnets, J.Appl. Phys. 49 (3) (1978) 1653e1658.

[44] J.F.LEffler, J.P.Meier, B. Doudin, J.-P.Ansermet, W. Wagner, Random and exchange anisotropy in consolidated nanostructured $\mathrm{Fe}$ and $\mathrm{Ni}$ : role of grain size and trace oxides on the magnetic properties, Phys. Rev. B 57 (5) (1998) 2915 e2924.

[45] J. Tejada, B. Martinez, A. Labarta, R. Grøssinger, H. Sassik, M. Vazguez, et al., Phenomenological study of the amorphous $\mathrm{Fe}{ }_{80} \mathrm{~B}_{20}$ ferromagnet with small random anisotropy, Phys. Rev. B 42 (1) (1990) 898 e905.

[46] R.S.Iskhakov, S.V.Komogortsev, A.D. Balaev, L.A. Chekanova, Dimensionality of a system of exchange-coupled grains and magnetic properties of nanocrystalline and amorphous ferromagnets, Pis'ma Zh. Eksp. Teor. Fiz. 72 (6) (2000) 440e444. JETP Letters 2000;72(6):304-7.

[47] S.V. Komogortsev, R.S. Iskhakov, A.D. Balaev, A.G. Kudashov, A.V. Okotrub, S.I. Smirnov, Magnetic properties of $\mathrm{Fe}_{3} \mathrm{C}$ ferromagnetic nanoparticles encapsulated in carbon nanotubes, Fiz. Tverd. Tela 49 (4) (2007) 700 e703. Phys Sol State 2007;49(4):734-8.

[48] H.M. Lu, W.T. Zheng, Q. Jiang, Saturation magnetization of ferromagnetic and ferrimagnetic nanocrystals at room temperature, J. Phys. D Appl. Phys. 40
(2007) 320 e325

[49] J.P.Bucher, D.C.Douglass, L.A. Bloomfield, Magnetic properties of free cobalt clusters, Phys. Rev. Lett. 66 (23) (1991) 3052 e3055.

[50] I.M. Billas, A. Chatelain, W.A. de Heer, Magnetism from the atom to the bulk in iron, cobalt, and nickel clusters, Science 265 (1994) 1682 e1684.

[51] D.R. Linde, Handbook of Chemistry in Physics, 73rd Edition, CRC Press Boca Raton, 1992.

[52] C.A. Ross, M. Hwang, M. Shima, J.Y.Cheng, M. Farhoud, T.A. Savas, et al, Micromagnetic behavior of electrodeposited cylinder arrays, Phys. Rev. B 65 (14) (2002), 144417-1-8.

[53] V.A. Bautin, A.G. Seferyan, M.S. Nesmeyanov, N.A. Usov, Magnetic properties of polycrystalline cobalt nanoparticles, AIP Adv. 7 (2017) 45103.

[54] S. Chikazumi, Physics of Ferromagnetis M, second ed., Oxford University Press, 1997.

[55] S. Vernon, S. Lindsay, M.B. Stearns, Brillouin scattering from thermal magnons in a thin Co film, Phys. Rev. B 29 (8) (1984) 4439e4442.

[56] G. Shirane, V.J. Minkiewicz, R. Nathans, Spin waves in 3d metals, J. Appl. Phys. 39 (2) (1968) $383 \mathrm{e} 390$

[57] R. Krishnan, FMR studies in compositionally modulated Co-Nb and Co films, J. Magn. Magn Mater. 50 (2) (1985) 189e192.

[58] C. Eyrich, W. Huttema, M. Arora, E. Montoya, F. Rashidi, C. Burrowes, et al., Exchange stiffness in thin film Co alloys, J. Appl. Phys. 111 (7) (2012), 07C9191-3.

[59] E. Girt, W. Huttema, O.N. Mryasov, E. Montoya, B. Kardasz, C. Eyrich, et al., A method for measuring exchange stiffness in ferromagnetic films, J. Appl. Phys. 109 (7) (2011), 07B765-1-3.

[60] M. Jamet, W. Wernsdorfer, C. Thirion, D. Mailly, V. Dupuis, P. Melinon, A. Perez, Magnetic anisotropy of a single cobalt nanocluster, Phys. Rev. Lett. 86 (20) (2001) 4676e4679.

[61] S. Oyarzún, A. Tamion, F. Tournus, V. Dupuis, M. Hillenkamp, Size effects in the magnetic anisotropy of embedded cobalt nanoparticles: from shape to surface, Sci. Rep. (2015), 14749-1-8.

[62] M.F. Hansen, S. Morup, Models for the dynamics of interacting magnetic nanoparticles, J.Magn. Magn Mater. 184 (1998) 262e274.

[63] M.A. Zaluska-Kotur, M. Cieplak, Dipole interaction with random anisotropy - a local-mean-field study, Europhys. Lett. 23 (1993) 85e90.

[64] S. Fleutot, G.L. Nealon, M. Pauly, B.P. Pichon, C. Leuvrey, M. Drillon, et al., Spacing-dependent dipolar interactions in dendronized magnetic iron oxide nanoparticle 2D arrays and powders, Nanoscale 5 (4) (2013) 1507 e1516.

[65] T. Wang, Y. Wang, Y. Fu, T. Hasegawa, T. Washiya, H. Saito, et al., In-field magnetic force microscope study of dipolar interaction in an ideally ordered Co nanorod array fabricated using nanoimprint lithography, Appl. Phys. Lett. 92 (19) (2008), 192504-1-3.

[66] F. Vidal, Y. Zheng, P. Schio, F.J. Bonilla, M. Barturen, J. Milano, et al., Mechanism of localization of the magnetization reversal in $3 \mathrm{~nm}$ wide Co nanowires, Phys. Rev. Lett. 109 (11) (2012), 117205-1-5.

[67] V. Russier, C. Petit, J. Legrand, M.P. Pileni, Collective magnetic properties of cobalt nanocrystals self-assembled in a hexagonal network: theoretical model supported by experiments, Phys. Rev. B 62 (6) (2000) 3910 e3916.

[68] D. Kechrakos, K.N. Trohidou, Dipolar interaction effects in the magnetic and magnetotransport properties of ordered nanoparticle arrays, J. Nanosci. Nanotechnol. 8 (6) (2008) 1e15.

[69] D. Sander, The correlation between mechanical stress and magnetic anisotropy in ultrathin films, Rep. Prog. Phys. 62 (1999) 809e858.

[70] B.D. Cullity, Introduction to Magnetic Materials, Addison-Wesley, Massachusetts, 1972.

[71] M.B. Stearns, Magnetic properties of 3d, 4d and 5d elements, alloys and compounds, in: Landolteb Grnstein Numerical Data and Functional Relationships in Science and Technology Group III, vol 19a, Springer, Berlin, 1986.

[72] C.H. Lee, H. He, F.J. Lamelas, W. Vavra, Cl.her, R. Clarke, Magnetic anisotropy in epitaxial Co superlattices, Phys. Rev. B 42 (1) (1990) 1066 e1069.

[73] A. Hubert, W. Unger, J.Kranz, Messung der Magnetostriktionskonstanten des Kobalts als Funktion der Temperatur [Measurement of the magnetostriction constants of cobalt as a function of temperature, Z. Phys. 224 (1969) 148e155.

[74] P. Bruno, Magnetic surface anisotropy of cobalt and surface roughness effects within Neel's model, J.Phys. F Met. Phys. 18 (1988) 1291e1298.

[75] A.F. Goncharov, J. Crowhurst, J.M. Zaug, Elastic and vibrational properties of cobalt to 120 GPa, Phys. Rev. Lett. 92 (11) (2004), 115502-1-5.

[76] G. Steinle-Neumann, L. Stixrude, R.E.Cohen, First-principles elastic constants for the hcp transition metals Fe, Co, and Re at high pressure, Phys. Rev. B 60 (2) (1999) 791e799.

[77] M.F. Yu, O. Lourie, M.J. Dyer, K. Moloni, T.F. Kelly, R.S. Ruoff, Strength and breaking mechanism of multiwalled carbon nanotubes under tensile load, Science 287 (5453) (2000) 637 e640.

[78] http://nmag.soton.ac.uk/nmag/.

[79] R.D. Gomez, M.C. Shih, R.M.H. New, R.F.W. Pease, R.L. White, Switching characteristics of submicron cobalt islands, J.Appl. Phys. 80 (1996) 342e346. 